\title{
Teaching Adult Rats Spinalized as Neonates to Walk Using Trunk Robotic Rehabilitation: Elements of Success, Failure, and Dependence
}

\author{
OUbong I. Udoekwere, ${ }^{1}$ Chintan S. Oza, ${ }^{2}$ and Simon F. Giszter ${ }^{1}$ \\ ${ }^{1}$ Neurobiology and Anatomy, Drexel University College of Medicine, Philadelphia, Pennsylvania 19129, and ${ }^{2}$ Department of Bioengineering, Duke \\ University, Durham, North Carolina 27708-0281
}

Robot therapy promotes functional recovery after spinal cord injury (SCI) in animal and clinical studies. Trunk actions are important in adult rats spinalized as neonates (NTX rats) that walk autonomously. Quadrupedal robot rehabilitation was tested using an implanted orthosis at the pelvis. Trunk cortical reorganization follows such rehabilitation. Here, we test the functional outcomes of such training. Robot impedance control at the pelvis allowed hindlimb, trunk, and forelimb mechanical interactions. Rats gradually increased weight support. Rats showed significant improvement in hindlimb stepping ability, quadrupedal weight support, and all measures examined. Function in NTX rats both before and after training showed bimodal distributions, with "poor" and "high weight support" groupings. A total of $35 \%$ of rats initially classified as "poor" were able to increase their weight-supported step measures to a level considered "high weight support" after robot training, thus moving between weight support groups. Recovered function in these rats persisted on treadmill with the robot both actuated and nonactuated, but returned to pretraining levels if they were completely disconnected from the robot. Locomotor recovery in robot rehabilitation of NTX rats thus likely included context dependence and/or incorporation of models of robot mechanics that became essential parts of their learned strategy. Such learned dependence is likely a hurdle to autonomy to be overcome for many robot locomotor therapies. Notwithstanding these limitations, trunk-based quadrupedal robot rehabilitation helped the rats to visit mechanical states they would never have achieved alone, to learn novel coordinations, and to achieve major improvements in locomotor function.

Key words: locomotion; motor cortex and spinal cord plasticity; recovery of function; robot rehabilitation; trunk control; voluntary and reflex integration

\section{Significance Statement}

Neonatal spinal transected rats without any weight support can be taught weight support as adults by using robot rehabilitation at trunk. No adult control rats with neonatal spinal transections spontaneously achieve similar changes. The robot rehabilitation system can be inactivated and the skills that were learned persist. Responding rats cannot be detached from the robot altogether, a dependence develops in the skill learned. From data and analysis here, the likelihood of such rats to respond to the robot therapy can also now be predicted. These results are all novel. Understanding trunk roles in voluntary and spinal reflex integration after spinal cord injury and in recovery of function are broadly significant for basic and clinical understanding of motor function.

\section{Introduction}

Spinal cord injuries (SCIs) are usually clinically incomplete (Dietz and Curt, 2006; Fitzgerald and Fawcett, 2007). Much SCI

Received June 6, 2014; revised June 5, 2016; accepted June 10, 2016.

Author contributions: U.I.U. and S.F.G. designed research; U.I.U. and C.S.O. performed research; U.I.U. and S.F.G. analyzed data; U.I.U., C.S.O., and S.F.G. wrote the paper.

This research was supported by National Institutes of Health Grants NS54894 and NS72651, and the Craig H. Neilsen Foundation. We thank Kavon Noorbehesht, Lollise Mbi, Katie German, and Drexel ULAR staff for excellent animal care; and the Drexel Spinal Cord Group for histological aid and support.

The authors declare no competing financial interests.

Correspondence should be addressed to Dr. Simon F. Giszter, Neurobiology and Anatomy, Drexel University College of Medicine, 2900 Queen Lane, Philadelphia PA 19129. E-mail: sgiszter@drexelmed.edu. research uses incomplete models, but complete SCI models provide "worst case" scenarios. Robot rehabilitation has advanced in both incomplete and complete animal models (de Leon and Acosta, 2006, de Leon et al., 2002; Musienko et al., 2012; van den Brand et al., 2012; Horst et al., 2013) together with methods promoting hindlimb stepping in complete SCI (Cha et al., 2007; Boyce et al., 2012; Gad et al., 2013; Ziemlińska et al., 2014). However, quadrupedal robot rehabilitation in animal models of complete SCI has been rare (Giszter et al., 2005; Udoekwere et al., 2006, 2014; Dominici et al., 2012). Here we test robot rehabilita- 
tion of neonatally spinal transected (NTX) rats during quadrupedal locomotion in adulthood and the successes and limitations of this.

Surprisingly, some neonatally spinalized rats $(\sim 20 \%)$ and cats master quadrupedal weight-supported locomotion into adulthood (Stelzner et al., 1975; Howland et al., 1995; Giszter et al., 1998), although they lack direct neural links between lumbar spinal cord and the voluntarily controlled CNS. Understanding how to promote such weight support in the other NTX rats may help designing rehabilitation for complete SCI. We have analyzed coordinative mechanisms and spinal and cortical features enabling NTX weight support (Giszter et al., 1998, 2007, 2008a, b). A "wheelbarrow race" or "pantomime horse" model of function nicely describes NTX rats weight support performance despite complete transection (Giszter et al., 2010): trunk actions coordinate the two independent neural control systems using the coupled biomechanics fore and aft (Giszter et al., 2007, 2008b) similar to a children's "wheelbarrow race." This coordination is likely a cortical mediated skill: there is a 1:1 relationship of specific trunk representations in motor cortex with higher function (Giszter et al., 1998, 2008a); lesion of these representations and trunk somatosensory overlap cortex abolishes high function (Giszter et al., 1998, 2008a); and specific biomechanical coordination associates with function (Giszter et al., 2007). Can these skills be taught to spinal transected rats? We hypothesized that some NTX rats that failed to develop quadrupedal weight support could subsequently learn this skill as adults, aided by robot rehabilitation. The rehabilitation supports the wheelbarrow control strategy through trunk training. We tested this using a pelvic orthosis (see Udoekwere et al., 2014). Robot rehabilitation was applied using the pelvic orthosis during quadrupedal treadmill locomotion. We used impedance based techniques of Krebs and Hogan from clinical rehabilitation (Krebs et al., 1998, 1999, 2008; Volpe et al., 2000, 2001, 2002, 2003, 2005). The results show significant functional improvements. Further, some nonweight-supporting NTX rats improved qualitatively and effectively transitioned to the weight-supporting category over training. These rats retained this function gain even after robot actuators were turned off. However, there was no retention with robot disconnected from the rats, overground, or in the home cage. The implications of these different elements of success and failure may be of broad relevance to rehabilitation processes.

\section{Materials and Methods}

Humane treatment and Institutional Animal Care and Use Committee approvals. All experimental procedures complied with the guidelines of the National Institute of Health Guide for the Care and Use of Laboratory Animals, USDA regulations, and received Drexel University Institutional Animal Care and Use Committee approval.

Surgery and preparation. Female Sprague Dawley rats received a complete spinal cord injury at T9/T10 at postnatal day 5 or 6 (NTX rats) (Fig. $1 C)$. Eighteen such rats used here received robot impedance sensorimotor treadmill training of trunk using an implanted pelvic orthosis (referred to henceforth as robotic rehabilitation training). Seven more rats served as treadmill-only trained controls, without robot training, but with similar implanted pelvic orthoses. Finally, 38 additional rats served as nonimplanted controls that received low-intensity treadmill training. These two control groups controlled for possible spontaneous changes in function in high or low function NTX rats similar to those we observed to occur in robot therapy. The first 7 rats controlled for spontaneous changes as a result of orthoses surgery and intense treadmill training alone. The second 38 rats formed a large control group, which served to guarantee that there were no spontaneous large changes in NTX rats weight support levels during maintenance treadmill training. It should also be noted that no such major changes have been observed in our laboratory previously (e.g., Oza and Giszter, 2015), or reported in any published literature.

With the exception of anesthetic method (we used isoflurane as opposed to hypothermia), spinal transection surgery is largely as described in detail in Miya et al. (1997) and Giszter et al. (1998, 2008a). Neonates were placed under isoflurane anesthesia and maintained on an anesthetic plane $(0.75 \%-2 \%$ mixed in oxygen), and sterile procedures were used throughout the surgery. For spinal transections, one complete segment of mid/low-thoracic spinal cord ( $\sim \mathrm{T} 9 / \mathrm{T} 10)$ was completely aspirated, and the cavity was filled with gelfoam. Postoperatively, all spinalized rat pups received analgesia during initial recovery comprising $0.01 \mathrm{ml}$ of $0.002 \mathrm{mg} / \mathrm{ml}$ buprenorphine subcutaneously. Thereafter, they were returned to their mothers until they reached $\sim 28 \mathrm{~d}$ of age, at which time they were weaned from the dams, and raised for another $28 \mathrm{~d}$ before initial testing. Following weaning, they were housed with other NTX rats (age-matched) and were regularly monitored to ensure continued thriving and development away from their dams

Early treadmill training and preliminary recovery assessments. All rats were treadmill-trained after weaning (at $\sim 2$ months postpartum) to maintain any early developmental achievements of some functional locomotor recovery and to optimize functional outcome before any robotic rehabilitation training (Fig. 1C). Treadmill exercise comprised 5-10 min locomotor training occurring 2-3 times a week on a motorized treadmill (speed $4-8 \mathrm{~cm} / \mathrm{s}$ ). Treadmill exercise sessions were periodically videotaped to assess the animals' achieved locomotion. We classified the animals' locomotion into one of two categories: (1) weight-supporting (WS) or 2) non-weight-supporting (NWS). This was accomplished in a manner similar to Miya et al. (1997) and Giszter et al. (1998) by determining the proportion of autonomous hindlimb weight-supporting steps (\%WSS) taken compared with the sum of hindlimb steps attempted (i.e., sum of weight-supported and non-weight-supported steps) (Miya et al., 1997; Giszter et al., 1998). The classification we used here was based on observations (Giszter et al., 2008a; see Materials and Methods) that most NTX rats showed either good consistent weight-supported stepping of $>50 \%$ WSS (weight-supporting NTX rats or WS, usually $\sim 20 \%$ of rats prepared) or poor weight-supported stepping of $<50 \% \mathrm{WSS}$, which was often accompanied with frequent hindlimb scissoring action (NWS) (Giszter et al., 2008a) (usually $\sim 80 \%$ of rats prepared). In prior work, the WS and NWS rats generated after neonatal spinal transection differed in a range of ways, with a "no-man's land" between the function levels of 20\%-25\%WSS and $45 \%-50 \%$ or better WSS (Miya et al., 1997; Giszter et al., 1998, 2007). Of the 18 NTX rats prepared for robotic rehabilitation training, 4 (22\%) were considered WS and 14 (78\%) were considered NWS. In the 38 nonimplanted control rats treated with light treadmill training, we observed $(9 / 38=24 \%)$ were WS and $(29 / 38=$ $76 \%$ ) were NWS rats. Thus, together, of the total 56 NTX rats prepared, we found $13(23 \%)$ were WS and $43(77 \%)$ NWS. These proportions of WS and NWS match many prior published data for such NTX rats from our laboratory and others (Stelzner et al., 1975; Miya et al., 1997; Giszter et al., 2008a). The large sample size and relative stability of proportions between iterations of surgeries give statistical force to the later effects of robot training that we observed. For the 7 additional matched treadmilltrained and implanted control rats, we selected only the NWS rats prepared similarly, specifically to control for the possibility of late spontaneous transition to weight support in treadmill training alone (see below). Prior work in our group suggests that treadmill training maintains WS levels of function in NTX WS rats but does not cause major improvements beyond that seen shortly after weaning. Here we explicitly examined large control groups of NTX NWS rats for any signs of spontaneous improvements.

Pelvic orthosis implantation surgery and postimplantation treatment. At $\sim 8-12$ months of age, the NTX rats for robot $(N=18)$ and treadmill only control rehabilitation $(N=7)$ were surgically implanted with a pelvic orthosis in the manner described by Udoekwere et al. (2014). Prior experience from our laboratory suggests successful integration of the orthosis with the bone in NTX rats is best achieved if implantation is performed at least 6-8 months after neonatal spinal cord injury, when the bone density in the haunches of the NTX rat is sufficiently high, despite the lower loading of hindlimbs in such rats. Udoekwere et al. 


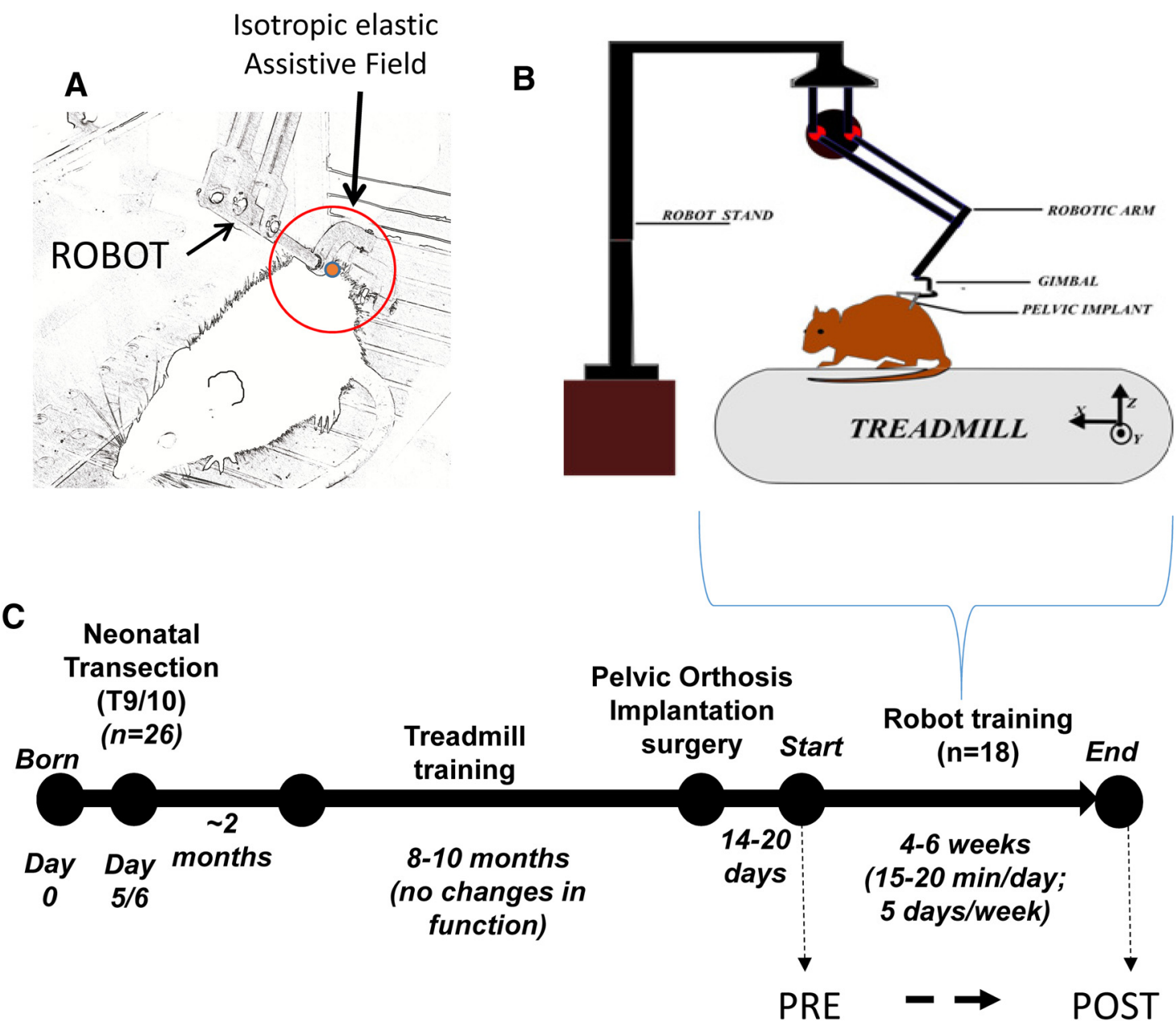

Figure 1. Robotic rehabilitation system and experimental and training timeline. $A-C$, Illustrations of robotic rehabilitation system showing cantilevered haptic phantom robotic arm connected via a gimbal to pelvic orthosis implanted in a rat $(\boldsymbol{A}, \boldsymbol{B})$ and isotropic elastic force field $(\boldsymbol{A}$, red circle) applied by robotic arm when connected to a rat while on treadmill during a training session $(\boldsymbol{A}$, B). For more detail on robotic rehabilitation training system, see Udoekwere et al. $(2006,2014)$ and Oza and Giszter (2014). C, Timeline of the experiment.

(2014) described the detailed pelvic orthosis design and surgical implantation and also demonstrated that the implant and surgery do not disrupt locomotion kinematics (Udoekwere et al., 2014). The implantation involved separating the gluteus muscle bilaterally with minimal tissue damage and then inserting each half of an orthosis such that it clamps to the iliac processes of rat's pelvis. Each half of the orthosis extends to a hollow bar outside the animal's body, which serves to support the gimbal attachment of the robotic arm (Fig. $1 A, B$ ). We have previously used this method extensively in adult spinalized rats and in intact rats (Udoekwere et al., 2006, 2014; W. Song et al., 2009; W. Song and Giszter, 2011; Hsieh and Giszter, 2011; Oza and Giszter, 2014). A total of 38 NWS NTX rats without pelvic implants served as additional controls. It should also be noted here that the \%WSS measures (see below) of all NTX rats were evaluated initially both before and after pelvic implantation, and were not significantly different. The pelvic implantation process did not significantly disrupt or change the preimplant hindlimb \%WSS performance in the NTX rats used for this study.

Robotic rehabilitation and treadmill training. Approximately $14-20 \mathrm{~d}$ following pelvic implantation surgery, NTX rats began robotic rehabilitation training similar to that described by Udoekwere et al. (2006) and Hsieh et al. (2011) (Fig. 1C). A cantilevered haptic phantom robot (1.0 scale, SenSable Devices) was connected via a gimbal to the implanted pelvic orthosis on the rat (Fig. $1 A, B$ ). During treadmill training, the robot applied an isotropic elastic force field (stiffness of $45 \mathrm{~N} / \mathrm{m}$ ) through the implanted orthosis such that the forces act to support and stabilize the pelvis/mid-low trunk region of the rat on an as needed basis while allowing it to step quadrupedally (Fig. $1 A$ ). The rationale for this training regimen design is the observation that the $20 \%$ of NTX of rats that achieve weight support spontaneously seem to rely on the trunk coupling and control of trunk to manage their improved function (Giszter et al., 1998, 2007, 2008a, 2010; Udoekwere et al., 2006). Equilibrium position for elastic force fields was set approximately at the average normal carriage height of an adult intact rat such that the trunk posture of NTX rat at which forces would be zero was similar to that of a normal rat. Because the typical carriage height of a non-weight-supporting NTX rat is de facto lower than that of a normal rat, the equilibrium position provided vertical elastic force that lifts such a rat and provides some weight support force at the haunches. Additionally, such NTX rats were still able to contact the treadmill surface (i.e., they do not "air step" when connected to the robot) and thus they experienced hindlimb loading while being positioned in a more "natural" trunk posture during training. This brings the NTX rats' pelvis into the approximate kinematic relationship needed for functional locomotion. The use of an elastic force field approach in our robotic rehabilitative setup is similar to the impedance training approach used for upper limb motion rehabilitation in stroke patients (e.g., using the MIT-MANUS) (Krebs et al., 1998, 1999, 2008; Volpe et al., 1999; Hogan and Krebs, 2004).

NTX robot-trained rats were trained with the robot system on the treadmill for $15-20 \mathrm{~min} / \mathrm{d}, 5 \mathrm{~d} /$ week for 4 weeks. Training sessions were periodically video recorded for assessment of function. The 15-20 min sessions constitute what physical therapists term "massed" training, and this was readily achieved with our robotic system. The 29 control NWS NTX treadmill rats were similarly prepared without 
implants, and either received daily massed training, or twice weekly treadmill training, over similar intervals.

Post-training and through training assessments of hindlimb locomotor recovery. Hindlimb locomotor function of the NTX rats through and after training was evaluated using the following 4 types of measures:

- Antri, Orsal, and Barthe (AOB) scoring scale for evaluating motor recovery of hindlimb gait coordination and stepping (Antri et al., 2002).

- Body weight support (\%BWS) provided by vertical robotic interaction forces.

- Proportion of autonomous weight-supported hindlimb steps taken on treadmill (\%WSS).

- Hindlimb joint kinematics.

All recovery outcomes measures (AOB, \%BWS, \%WSS, and kinematics) were first evaluated during robotic rehabilitation training while the robot was connected to the animal and the elastic field was active. The $\%$ WSS measure was the primary measure used to assess functional recovery without the robot connected before any robotic rehabilitation training and at the end of the training period because the \%WSS measure is the basis of the bimodal distribution seen in NTX rats. This allowed for comparison between nonrobotic pretraining \%WSS performance and nonrobotic post-training \%WSS performance.

Hindlimb motor recovery score. Hindlimb motor recovery of hindlimb local gait coordination and stepping was assessed from recorded video using a scale designed by the AOB scale, which is a modified BBB-type scale for hindlimbs only (Antri et al., 2002). Video was collected at $60 \mathrm{~Hz}$, $1 / 500 \mathrm{~s}$ shutter during each robotic rehabilitation training session. An angled mirror placed under the treadmill was used to capture footpad placement during video recording. The AOB scale scored rats based on frequency, amplitude, and coordination of hindlimb stepping motions, presence of weight-supported stepping, and frequency of plantar stepping. The AOB scale ranged from 0 to 22, with a score of 0 representing no hindlimb locomotor recovery, and 22 representing significant hindlimb locomotor behavior akin to that of a normal intact rat. More specifically, scores of $0-1$ correspond to animals unable to support their body weight with their hindlimbs and unable to step. Scores of 2-9 correspond to rhythmic movements of hindlimbs without any body weight support. A score of 10 corresponds to consistent rhythmic movements with dorsal foot placement and occasional body weight support. Scores of 11-22 correspond to rhythmic movements of hindlimbs with plantar paw placement with the possibility of body weight support. All AOB scoring was done using video recorded during robotic rehabilitation training sessions, and the scores were used to assess the cumulative effect of robotic rehabilitation training session. A 3-5 min segment of the recorded video was used to assess AOB score every other training day.

Percentage of hindlimb WSS. We evaluated the number of hindlimb weight-supported steps taken during robotic rehabilitation training using the same recorded video segment used for AOB scoring. The total number of hindlimb weight-supported steps was expressed as a percentage of total hindlimb steps (i.e., sum of weight-supported and non-weight-supported steps) attempted (\%WSS). This technique for quantifying autonomous weight-supported stepping (\%WSS) was adopted from details by Miya et al. (1997). A hindlimb step was considered as a weight-supported step if the hindlimb was seen to be raised above the surface of the treadmill with no contact of the belly, or proximal hindlimb joints (knee or hip), with the treadmill during the swing and stance phases of the hindlimb step cycle. A step cycle was counted as a movement of a hindlimb that included ankle extension (typically during stance) and flexion (typically during swing). Typical weight support steps included successive stance and swing phases with hindquarter support. Plantar foot placement was not required and (knuckle walking) dorsal stepping was permitted. \%WSS was calculated by counting the number of weight support steps achieved, divided by the total steps attempted within the scoring interval. \%WSS assessment was collected every other training day. Assessing the autonomous weight-supported stepping allowed us to capture improvements in weight bearing capability over the training period.
We also determined the \%WSS on the treadmill before and after pelvic implantation before robotic rehabilitation training. \%WSS after pelvic implantation was tested after recovery from surgery (2-3 weeks), and before any robotic rehabilitation training began (i.e., the animal had had no exposure or experience with the robot or elastic field at this point). $\%$ WSS evaluation was done to assess any deterioration due to the pelvic implant surgery.

Finally, we evaluated the \%WSS on the treadmill in the passive robot configuration (robot-inactive test; i.e., robot attached to rat but without any active control or force field assistance) as well as the \%WSS off the robot (off-robot test; i.e., robot fully disconnected from the rat) on the last day of training.

Robot provided percentage of BWS. Animals were also evaluated by examining changes in \%BWS provided by the robot during robotic rehabilitation training. This was accomplished by recording and analyzing the changes in the vertical force interactions between the animal and the robot's elastic field. These were recorded during every training session at $1 \mathrm{kHz}$. The mean vertical interaction force for each training session was determined over the same video segments used for AOB and \%WSS assessment, and normalized to the animal's body weight to calculate the $\%$ BWS. \%BWS was determined every other training day alongside the $\mathrm{AOB}$ and \%WSS scoring. The \%BWS measure allowed assessment of assistive body weight support provided by the robot and thus reflected changes in the animal's weight bearing capacity over the course of training, and the consequent motion of the rats' pelvis toward a more normal height, and reduction of robot assistive forces.

Hindlimb joint kinematic gait analysis. The recovery impact of the robotic rehabilitation training was also evaluated by analyzing hindlimb gait kinematics in detail in a subset of NTX rats (which included both weight-supporting and non-weight-supporting NTX rats) to determine any changes in the range of motion (ROM) of the hip, knee, and ankle joints between the first and last days of training.

Our approach captured in detail 2D gait kinematics of the right hindlimb. The right hindlimb and pelvic region was shaved to improve the visual image obtained for analysis. Five 3- to 4-mm-diameter spherical reflective disks (B\&L Engineering) were placed on the skin of lateral side of right hindlimb overlaying the iliac crest, the greater trochanter, the knee joint, the lateral malleolus, and fifth metatarsal head. To minimize the effects of skin slippage over the knee, the "true" knee joint was computed from video by using the actual lengths of the femur and tibia and a triangulation algorithm using the hip and ankle makers. The markers on the rat were illuminated with two infrared light source (model S-1800, Pinecom) and a high-speed digital image camera (JAI TM$6710 \mathrm{CL}$ ) was used to record motion of the lateral side of the right hindlimb in the sagittal plane (10 in. $\times 13$ in.) at $100 \mathrm{frames} / \mathrm{s}$. Motion tracking-analysis software (Maxtraq 2D, Innovision Systems) and a numerical computing package (MATLAB R2008, The MathWorks) were used to compute joint kinematics. Treadmill speed was set at $8-10 \mathrm{~cm} / \mathrm{s}$ and 1-2 min satisfactory continuous walking trials were recorded for each rat on the first and last days of training.

Data analysis. At the commencement of robotic rehabilitation training, all NTX robot rehabilitation recipient rats were initially grouped into two categories: weight-supported NTX rats (WS, $>50 \%$ WSS) or nonweight-supported NTX rats (NWS, $<50 \%$ WSS). The 18 robot-trained rats in the study broke down into initial distributions of 4 WS and 14 NWS before the robotic rehabilitation training. However, at the end of the training period, some of the robot-trained NWS rats were highly improved and able to achieve $>50 \%$ WSS. This was never observed spontaneously in control groups. The likelihood of one, or several, of these recovery of function events having occurred by chance was estimated using the number of control animals tested, and assuming binomial probabilities based on these observations. The distributions of \%WSS remained bimodal after training. Therefore, using this postexperiment observation, we then, post hoc, further subdivided the NWS group into 2 subgroups: nontransitioning non-weight-supported NTX rats (NTWS), which did not gain sufficient significant weight support so as to switch \%WSS category; and transitioning non-weight-supported NTX rats (TWS), which switched category at the end of training. For this later post hoc analysis, therefore, rats were grouped according to these 3 categories: 
WS, TWS, NTWS. This post hoc classification allowed us to better investigate the differences in recovery rates and mechanisms associated with the robot training paradigm in the NTX rats, and explore whether those rats which changed category might be predicted in future work.

Statistical analysis. In-depth statistical analysis was performed using standard tools in SPSS. To analyze the impact of robotic rehabilitation training on functional recovery measures, two-way repeated measure ANOVAs among the NTX groups over the training period were performed, with time taken as a repeated measure. We avoided ANCOVA because the ANCOVA assumptions of linear trends in the repeated measures did not hold for the individual rats. Further, the repeated measures approach matches standard statistical methods used in many designs (Edwards, 1985; Portney and Watkins, 1993). If the ANOVA effect was significant, we then proceeded to post hoc tests with Bonferroni corrections, which were performed to identify specific group differences. Before using two-way repeated-measures ANOVAs, sphericity tests were used to check for normal distribution of the data for each recovery measure. If sphericity was violated, then Greenhouse-Geisser corrections were applied to the degrees of freedom to estimate sphericity. Post hoc tests were also used for appropriate significance corrections (e.g., Bonferroni).

A second analysis of recovery was used to examine individual differences in rate of recovery. Regressions of the recovery trends in the $\% \mathrm{BWS}$ \%WSS, and AOB measures were performed in the individual rats. The resulting slopes of these regressions were then used in a one-way ANOVA set of comparisons of recovery processes. However, it should be noted that we proceeded in this way because of the form of some of the recovery data. We observed that only the TWS rats were somewhat sigmoidal in their recovery processes in the WSS and AOB measure,. These sigmoidal recovery processes prevented straightforward use of more principled omnibus statistical methods, such as ANCOVA for trend variations in our rats. The \%WSS slope measures of the simple linear fits in individual rats represented very crude measures of their improvement in performance. Late sigmoids and early sigmoids had differing linear fit slopes, thus capturing aspects of the rate and timing of recovery, albeit crudely. These analyses with regression slopes therefore support limited interpretations about individual recovery speeds, as noted in Results.

Significance levels were set at $p<0.05$ for all comparisons. Statistics were prepared using the SPSS package and MATLAB computational software (The MathWorks).

Histological confirmations and methods. NTX rats were deeply anesthesized with $3 \mathrm{ml}$ of Euthasol following robotic training and assessments, and perfused intracardially with $0.9 \%$ physiological saline followed by $4 \%$ buffered PFA to fix spinal tissue. Spinal cord tissue was extracted, and blocks containing segments rostral and caudal to the transection were prepared for cryostat sectioning. Blocks rostral and caudal to the spinal transection were cut in serial transverse $20 \mu \mathrm{m}$ sections, and blocks containing the lesion were cut in serial parasagittal $20 \mu \mathrm{m}$ sections. Nissl myelin and serotonin immunohistochemical stains with DAB were then used to examine the completeness of spinal injury. All rats were confirmed to be histologically complete spinal transections with no spared tissue at the transection and no serotonin staining below the transection.

\section{Results}

The goal of this study was to evaluate the effect of robotic rehabilitation training (trunk impedance sensorimotor training) on locomotor recovery of adult rats spinalized as neonates (NTX rats). Eighteen rats were prepared for robot rehabilitation, all receiving complete spinal transection as neonates. Before robotic rehabilitation training, all 18 NTX rats received weekly treadmill training alone and during this period their \%WSS scores and function remained stable (4 WS, $22 \% ; 14$ NWS, $78 \%$ ). In other words, these robot-trained rats could be considered as acting as their own controls, by showing no significant performance changes over the extended time period before beginning their robot training. However, an additional 38 control rats were prepared. These were lightly trained on treadmill without pelvic implant or robot rehabilitation. These 38 control rats showed a similar distribution of WS $(9 / 38,24 \%)$ and NWS $(29 / 38,76 \%)$ rats to the initial distribution seen in the implanted experimental group rats when tested before robot rehabilitation. These rats thus acted as independent controls, and this unimplanted control group remained stable in their \%WSS measures over time with no spontaneous improvements (initial numbers 9 WS and 29 NWS; final numbers 9 WS and 29 NWS, with no switches of classification by any rats). Finally, a cohort of 7 additional rats were chosen because they were categorized as NWS. These controls were prepared with orthosis implants and trained intensively on treadmill. They were used post hoc as additional controls to compare treadmill training effects versus robot rehabilitation effects in the orthosis implanted NWS rats. The 7 treadmill-trained NWS control rats showed neither significant improvements nor decrements in their motor scores through training (mean \%WSS initial score 5\%, initial to final comparison: $p=0.244$; mean AOB initial score 4 , initial to final comparison: $p=0.45)$.

We examined three functional recovery measures in the robotic rehabilitation trained rats. These included the following: (1) a behavioral assessment score for hindlimb motor recovery and stepping function (AOB score); (2) the average degree of vertical weight support required of the robot rehabilitation system (\%BWS); and (3) the percentage of hindlimb steps that were fully weight-supporting (\%WSS). These measures all quickly improved over time in this group, and relative to the controls. We paid attention to these measures particularly in relation to the previously reported discontinuity in function and performance in WS and NWS rats. The 7 treadmill-trained NWS control rats did not show any significant functional improvements. The 38 long-term NTX control rats showed the following: (1) the distribution of function observed before training in the robot rehabilitation trained rats was not in any way atypical; it closely matched the control group; and (2) in the absence of robot training, the WS NWS classifications in the controls were stable over time. However, all robot rehabilitation rats showed significant improvement in all scores over time, and in comparing the final week of testing to the first.

\section{Effect of robot training on NTX rats as a group}

Plots of all robot-trained rats combined as a group and divided into WS and NWS rats over time are shown in Figure 2. For all 18 robotic trained NTX rats together, the hindlimb motor recovery as indicated in the AOB score versus training day plot (Fig. 2A; NWS + WS line) showed a trend of improved hindlimb motor performance that was highly significant (linear regression, $R^{2}=$ $0.89, p=3.7 \mathrm{e}-5)$. Furthermore, a paired $t$ test with Bonferroni correction showed that mean $\mathrm{AOB}$ scores on the first day of training were significantly different from the last day of training mean AOB scores $(p<0.001)$. That is, a significant AOB score improvement in the rats followed robot training. In contrast, control rats with intense treadmill training but no robot exposure showed no changes from first to last training days.

The quality of the rats' hindlimb stepping, as assessed by AOB, benefited significantly from our robot training at the pelvis, although the leg motion and interaction were not explicitly trained or controlled. Breaking out WS and NWS rats separately (WS line and NWS line; Fig. 2) showed that rats in both categories also improved significantly.

For all robot-trained rats together, the \%BWS trended down and was significantly reduced (linear regression $R^{2}=0.75, p=$ 9.9e-3) as shown in Figure 2B. This trend indicated that the rats moved their pelvis up (partially self-supported) and thereby reducing the body weight-supported assistance provided by the robot. Similar to the AOB results, a paired $t$ test with Bonferroni 
A

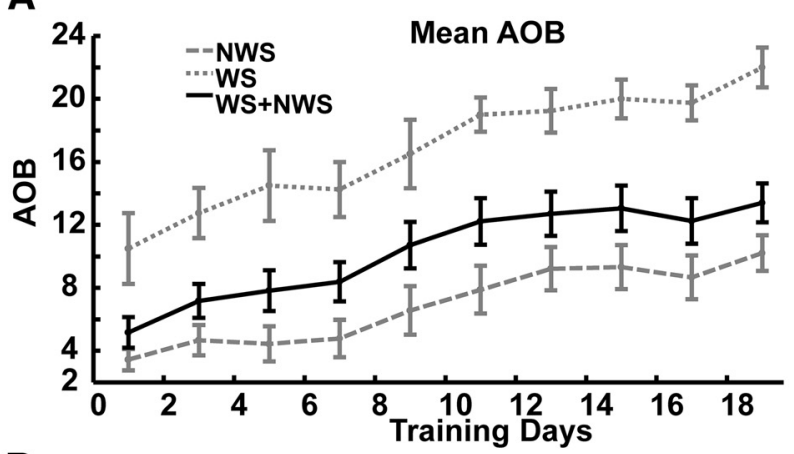

B
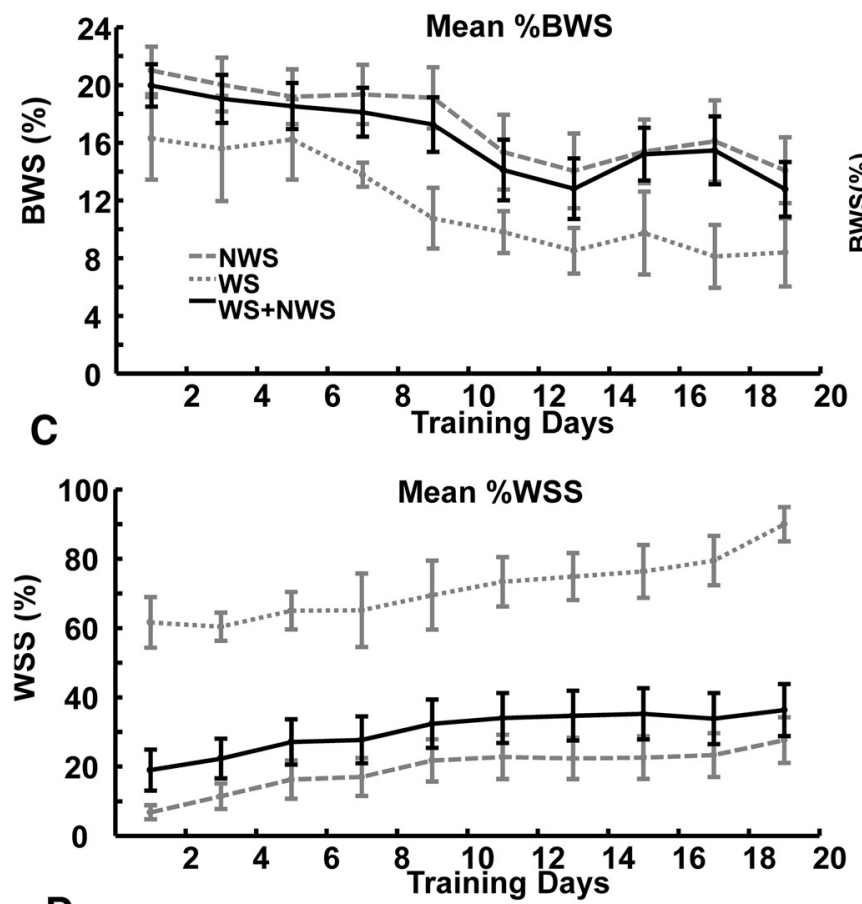

D

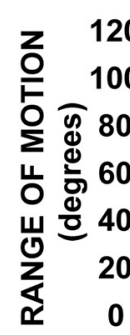

Mean AOB:

First and Last Training Days

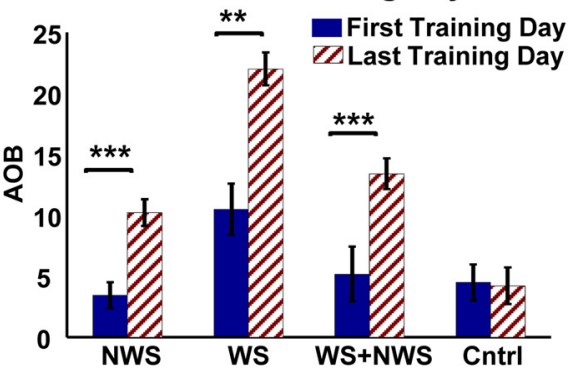

Mean \%BWS:

First and Last Training Days

First Training Day

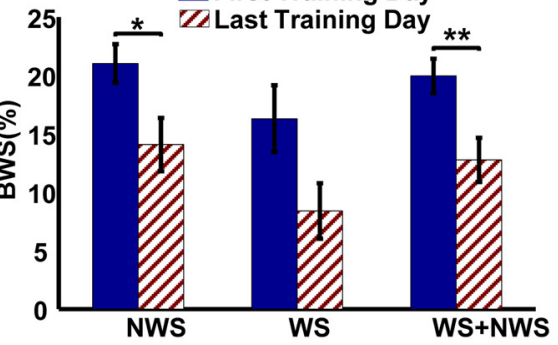

Mean \%WSS:

First and Last Training Days

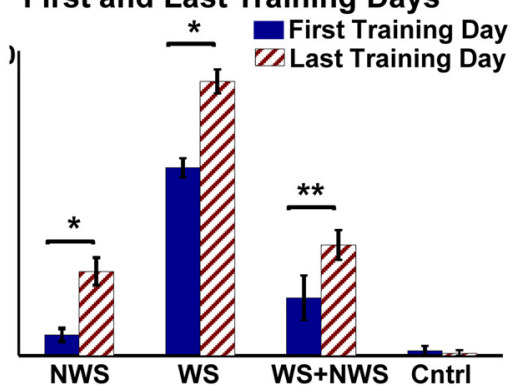

NWS

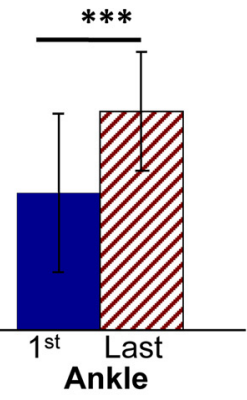

HINDLIMB JOINTS

Figure 2. Significant improvement in hindlimb motor performance (AOB score), reduction of body weight support provided by robotic assistance (\%BWS), increase in proportion of autonomous weight-supported hindlimb stepping (\%WSS) in the NWS group of 14 adult rats spinalized as neonates (NTX rats) and WS group of 4 NTX rats, as well as the two groups combined (NWS + WS) as 18 NTX rats following robotic rehabilitation training (trunk impedance sensorimotor treadmill training). $A$, Mean \pm SE plot of AOB scores for the NWS group, WS group, and combined group over the course of robot rehabilitation training (left), and bar plot (mean \pm SE) showing post hoc comparison of AOB scores between the first and last day of training for the same NWS, WS, and combined groups, as well as the control group of 7 matched treadmill only-trained NTX rats (right). Day 1 is considered the first day of training and day 19 is the last day of training. $\boldsymbol{B}$, Mean \pm SE plot of $\%$ BWS for the NWS group, WS group, and combined group over the course of robot rehabilitation training (left), and bar plot showing posthoc comparison of \%BWS between the first and last day of training for the NWS group, WS group, and combined group (right). Control rats shown in A, Chad no robot training and thus were not tested in this way. C, Mean \pm SE plot of \%WSS for the NWS group, WS group, and combined group over the course of robot rehabilitation training (left), and bar plot showing post hoc comparison of \%WSS between the first and last day of training for the same NWS, WS, and combined groups, as well as the control group of 7 matched treadmill only-trained NTX rats (right). Significant trends $\left(R^{2}>0.80,{ }^{* * *} p<0.001\right)$ of improved performance were observed in the AOB scores, \%BWS, and \%WSS for the NWS, WS, and combined (NWS + WS) groups over the course of training. The mean AOB, \%BWS, and \%WSS performances on the last day of training were significantly better than the first day of training for the NWS and for the combined group. ${ }^{* *} p<0.001 .{ }^{* *} p<0.01 .{ }^{*} p<0.05$. However, although improvements were seen in the \%BWS for the WS group, only the AOB and \%WSS performances on the last day of training were significantly better than the first day of training. D, Bar plots showing comparison of Range of Motion (ROM) of the hip, knee, and ankle joints during treadmill gait between the first and last day of training for subset of 6 NTX rats. There was a clear effect of training (two-way repeated-measures ANOVA, ${ }^{* * *} p<0.001$ ) on the increases seen in the ROM of the hip, knee, and ankle joints. Post hoc tests showed that the increases in ROM were significant in knee and ankle joints (**** $<<0.001 \mathrm{for}$ both) but not in hip ROM with the number of rats tested. 


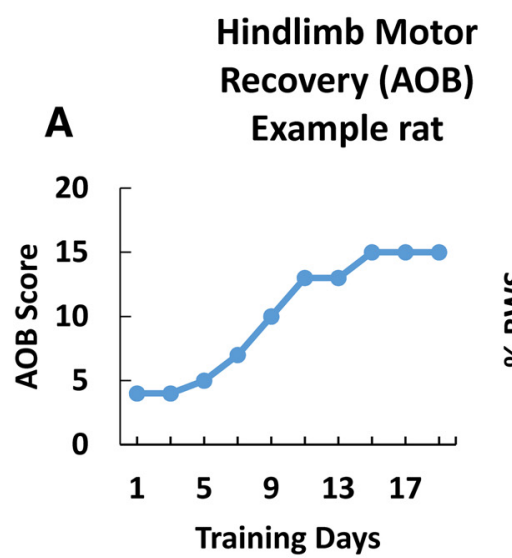

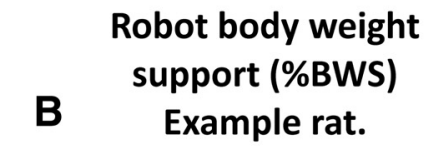

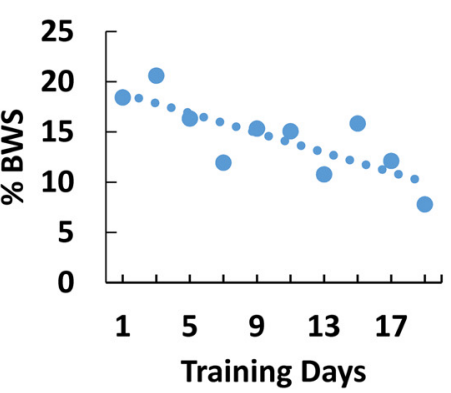

\section{\%Weight Supported \\ Steps (\%WSS)}
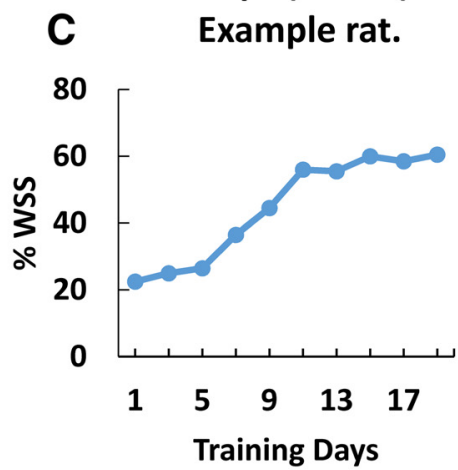

D1 Day1 of Robotic Training
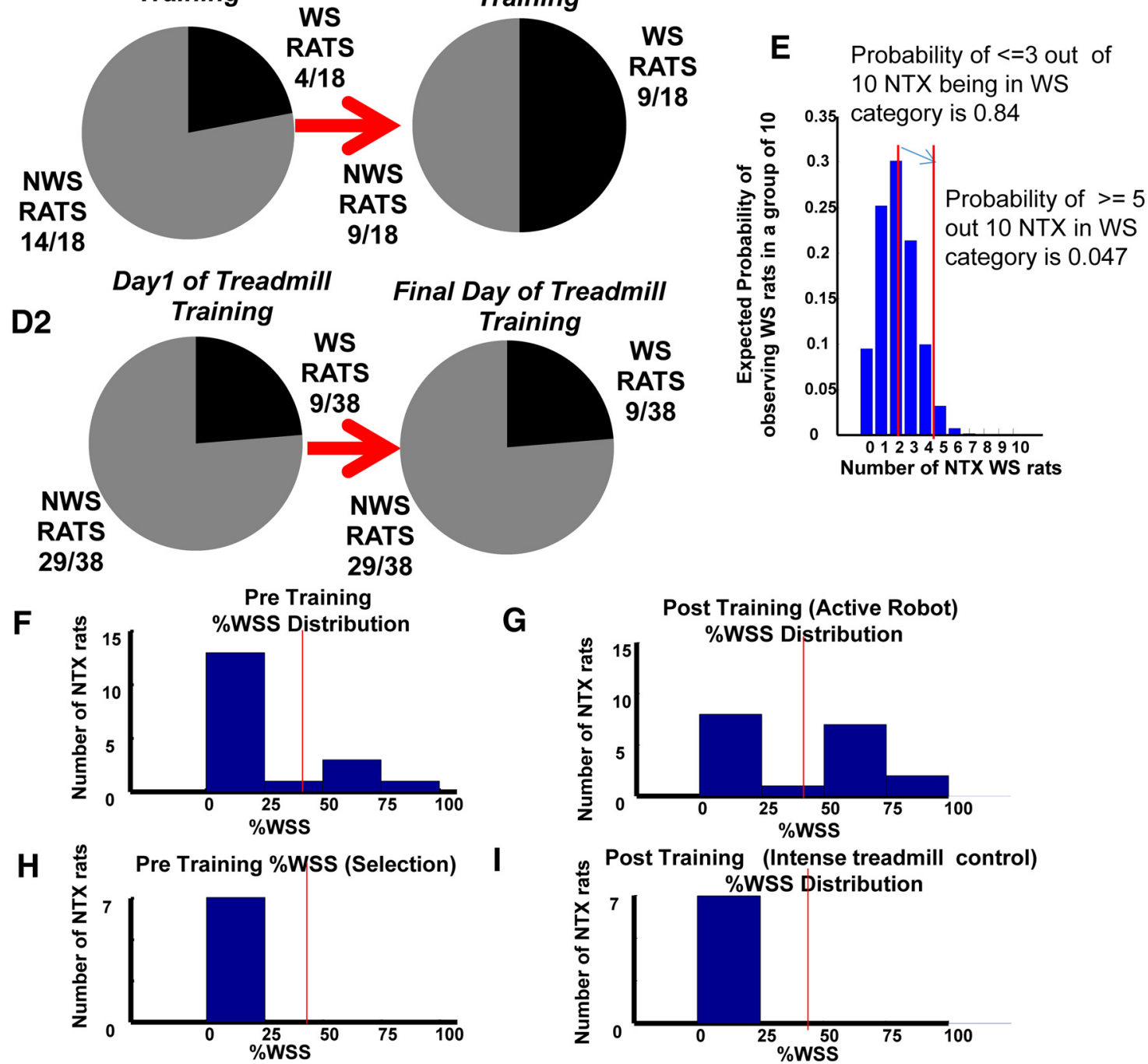

Figure 3. Some rats initially classified as non-weight-supporting before training with robotic rehabilitation training showed a transition to a weight-supporting classification. $A-C$, An example of $\mathrm{AOB}(\boldsymbol{A}), \% B W S(\boldsymbol{B})$, and \%WSS $(\boldsymbol{C})$ recovery progressions for a particular rat that transitioned from a non-weight-supporting category $(<50 \%$ WSS) to a weight-supporting category ( $>50 \%$ WSS) over the course of training. As shown in C, this particular rat had $<50 \%$ WSS on training day 1 and increased to $>50 \%$ WSS over the training period. A sigmoidal pattern of recovery progression is also seen in the $A 0 B$ and \%WSS measures $(\boldsymbol{A}, \mathbf{C}) . \mathbf{D 1}, 0 \mathrm{n}$ the first day of training, 14 of the 18 rats that received robotic rehabilitation training $(\sim 78 \%)$ were initially classified as non-weight-supporting before training, whereas the remaining 4 rats $(\sim 22 \%)$ were classified as weight-supporting. On the final day of training, 5 of the 14 non-weight-supporting rats transitioned to a weight-supporting category, thus increasing the proportion of rats in the WS classification from $22 \%$ to $50 \%$. Based on these observations, and to further understand the training effects of our robot rehabilitation, we grouped together these 5 originally non-weight-supporting rats that then transitioned to weight support with training as a TWS rat group $(n=5)$. The originally weight-supporting rats were grouped as WS rats $(n=4)$, whereas the originally non-weight-supporting rats that failed to transition with training were grouped as NTWS ( $n=9$ ). D2, In contrast to D1, there were no changes in weight support classifications following low-intensity treadmill-only training for any of the 29 non-weight-supporting and 9 weight-supporting implanted control rats on the last day of training. $\boldsymbol{E}, \mathrm{Z}$-score statistic for the last versus first training day distributions of weight-supporting (Figure legend continues.) 
correction showed that the first day mean \%BWS score was significantly different from last day of training \%BWS score ( $p=$ 0.003 ) indicating significant \%BWS improvement. Again, both WS and NWS rats benefited from the training. For all robottrained rats, the \%WSS trend line was significant (linear regression, $R^{2}=0.85, p=0.00016$ ) as shown in Figure 2C. Paired $t$ test with Bonferroni correction showed that first day mean WSS score was significantly different from the last day of training \%WSS score $(p=0.006)$ indicating significant WSS improvement of the rats as a whole. Similar to Figure $2 A$, control rats with intense treadmill training but no robot exposure showed no such changes from first to last training days. Again, separating WS and NWS rat groups showed that both categories of \%WSS rats improved with robot training.

A subset of NTX rats (1 WS, 2 TWS, and 3 NTWS) were used to evaluate and compare ROM of the hip, knee, and ankle joints during gait between the first and last days of training. Figure $2 D$ shows an ROM comparison of the first and last training days for each hindlimb joint. A two-way repeated-measures ANOVA of the hip, knee, and ankle ROM on the first and last day of training also showed a clear effect of robot training on kinematics on the treadmill between the first and last day on range of motion and significant increase in $\operatorname{ROM}\left(F_{(1,51)}=39.96, p<0.001\right)$. Post hoc tests showed the effect of robot training on increases in ROM were significant in knee and ankle joints $(p<0.001$ for both) but not in hip ROM with the number of rats tested.

In summary, examining the robot-trained rats as a combined group showed a significant positive impact of pelvis centered robot therapy on all our measures of function.

\section{Some NWS rats transitioned across the "no-man's land" to the weight-supported category}

In prior work, the WS and NWS rats generated after neonatal spinal transection differed in a range of ways, with a "no-man's land" between 20\%-25\%WSS (NWS) and 45\%-50\%WSS (WS) (Miya et al., 1997; Giszter et al., 1998, 2007). This distribution was clearly also present here in both the robot rehabiltation group (see Fig. $3 F$, histogram and vertical line) and also in the group of 38 age-matched and treadmill maintenance NTX controls.

\section{$\leftarrow$}

(Figure legend continued.) rats $\left({ }^{*} p<0.05\right)$ and a simulated distribution of expected numbers of NTX WS in a sample of 10 rats selected by chance from NTX rats based on a $20 \%$ chance of WS in the NTX population. The number of rats that transitioned from non-weight support to weight support categories increased the number of weight support classified rats at the end of training by far greater than could be expected by chance in a random sampling of a typical binomial distribution of neonatal transected rats. In 18 NTX rats, after training 9 were WS (i.e., $5 / 10$ ). The probability of $5 / 10$ rats by chance is 0.04 (i.e., $p<0.05$ ). Based on the $\mathbf{D 2}$ distribution, the probability of observing 9 of 18 rats to be in the WS category by chance is 0.009 . Further, we know the original distribution before training was typical and closely resembled an independent control group of 38 NTX rats in D2, all of which remained stable over time. The data are consistent with and support an accelerated \%WSS improvement seen in the TWS group that facilitated transition from a non-weight-supporting classification to a weight-supporting classification with training. $\boldsymbol{F}$, Histogram showing \%WSS distribution for all robot-rehabilitated NTX rats before robotic training. Vertical line indicates the separation of WS and NWS rats with a "no man's land" of low likelihood between. G, Histogram showing \%WSS distribution for all robot-rehabilitated NTX rats after training (robot-active) TWS rats moved to $>50 \%$ and thus increase the number of WS and reduce the NWS number compared with the initial pretraining distribution shown in $\boldsymbol{F}$ for the same animals. $\boldsymbol{H}$, Histogram showing \%WSS distribution for the control group of 7 matched treadmill-only trained NWS NTX rats before treadmill training. Vertical line indicates the separation of WS and NWS rats. I, Histogram showing \%WSS distribution for the control group of 7 matched treadmill-only trained NTX rats after training. In the 7 NTX NWS-matched control rats, there is no change in \%WSS distribution following treadmillonly training similar to the training intensity of the robot-rehabilitated NTX rats.
When examining \%WSS changes in individual rats over the course of robotic rehabilitation training here, we observed that some rats ( 5 from the initial 14 NWS) transitioned from a NWS classification ( $<50 \%$ WSS $)$ to a WS classification ( $>50 \%$ WSS $)$ while on the robot, effectively crossing over this \%WSS "noman's land" that exists in NTX rats (Miya et al., 1997; Giszter et al., 1998), as a result of training on the robot. Some qualitative change in overall function appeared to separate these groups. We also observed that the improvements seen in the AOB and \%WSS measures of these rats over the course of training were typically sigmoidal, as shown in an example rat in Figure $3 A$, $C$. These transition rats showed significant improvements in their \%WSS measures that were sufficient for us to reclassify them as now falling into the WS category at the end of robot training period (Fig. 3A-C). No control rats showed such changes (see below). The number of robot-trained rats in the WS category and NWS category are shown as a fraction of the robot-trained rats in Figure 3D1, for the first day and last day of training. Many more rats than could be expected by a chance selection were in the WS category after training (the $z$-score statistic for the last vs first training day distributions: $p<0.05$; Fig. $3 E$ ). Further, there were no such changes in function or movement away from the NWS class in any of the rats in either control group. The light treadmill alone control group is shown in Figure 3D2: the NWS number $N=29 / 38$ remained stable over lengthy training. Distributions of rats selected for intense treadmill + orthosis training over 4 weeks are shown in Figure $3 H, I$. Both before and after distributions, NWS number was stable $(N=7)$. However, the number of rats with large improvement that we found crossing between the NWS and WS categories following robot training was significant (Fig. 3D1, F, H). These increased the number of WS classified rats at the end of training to levels far greater than could be expected by chance in a typical sampling of neonatal transected rats (Fig. $3 E$ ). Similarly, the number was significantly different from that expected for a random resampling from a large population, which directly matched with the initial distribution of WS rats that we actually observed. Finally, because we saw no variations causing movement into the WS classification over time in either of the two control groups' NWS rats (when combined 36 NWS rats), it follows that the transition of some NWS rats to WS levels of weight support classification in the robot-trained group was a priori extremely improbable. (For example, assuming an [unobserved] probability of a transition event of $1 / 29$, or 0.034 , because none of 29 control rats observed showed any transitions, then the probability of observing 5 rats transition events in data as seen here has $p<10^{-7}$ ). It should not have occurred either spontaneously, or by chance, based on the control groups' distributions and the stability of these over time. As a result of the robot rehabilitation, some rats thus showed clearly significant qualitative as well as quantitative improvements in function.

Based on these observations, and to further understand the training effects of our robot rehabilitation, we post hoc divided the 18 robot-trained rats into 3 new categories or groupings: (1) originally weight-supporting (WS, $n=4$ ); (2) originally nonweight-supporting but transitioning with training to weight support (TWS, $n=5$ ); and (3) originally non-weight-supporting and then failing to transition with training to weight support (NTWS, $n=9$ ). Using these regroupings, we then analyzed the impact of robotic rehabilitation training on the different recovery measures. 


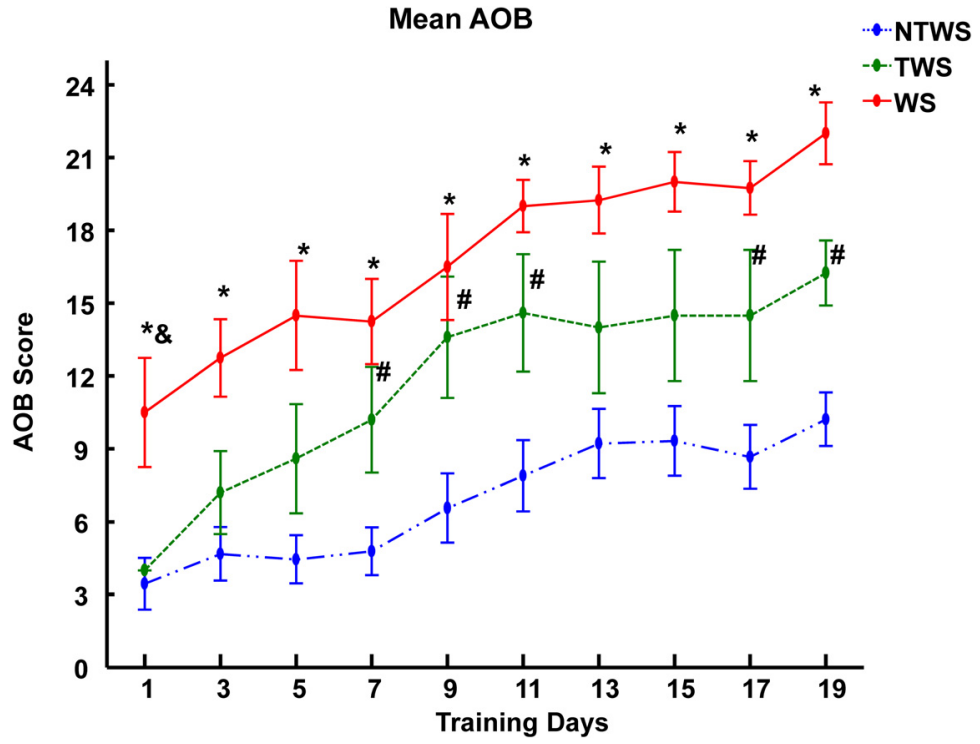

Figure 4. Robotic rehabilitation training improves hindlimb motor performance score (AOB score) in the WS, TWS, and NTWS groups. Plot represents mean \pm SE of $A O B$ score changes for each group over the course of robot rehabilitation training. There was a significant training effect (two-way repeated-measures ANOVA, $F_{(3.99,59.86)}=31.28,{ }^{* * *} p<0.001$ ) on AOB score over time in all three groups of rats. Post hoc comparisons of the training effect indicated that the AOB scores on the last training day were significantly greater than the first training day for each group ( $p<0.05$ for each group). There were also differences seen in the $A O B$ recovery performances among the groups as indicated by the group effect reported in the two-way repeated-measures ANOVA $\left(F_{(2,15)}=15.98,{ }^{* * *} p<0.001\right)$. Post hoc comparisons of the group effect showed that the mean AOB score for the NTWS group was significantly less than both the WS $(p<0.001)$ and TWS groups $(p<0.05)$, whereas the TWS group was not different from the WS group $(p=0.086)$. As shown in the figure, this is also evident in the daily post hoc comparisons among groups.

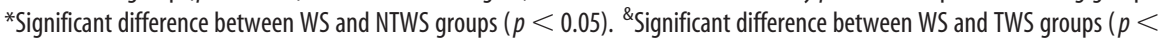
0.05). "Significant difference between TWS and NTWS groups $(p<0.05)$.

\section{AOB changes by group}

Figure 4 shows the $\mathrm{AOB}$ changes seen in each group following the regrouping. The AOB scoring scale was used to assess overall hindlimb motor recovery of gait coordination and stepping ability in NTX rats. Two-way repeated-measures ANOVA showed that there was a main effect of training over time on AOB performance $\left(F_{(3.99,59.86)}=31.28, p<0.001\right)$. This suggests that robotic rehabilitation training influenced hindlimb motor recovery in all 3 groups.

Post hoc tests of the training effect with Bonferroni corrections showed that the AOB performance on the last training day was significantly greater than the performance on the first training day for each group ( $p<0.05$ for comparison within each group). The ANOVA results also reported a significant group effect $\left(F_{(2,15)}=15.98, p<0.001\right)$. Post hoc analysis of the group effect with Bonferroni corrections revealed that the AOB performance of the NTWS group was significantly less than the WS $(p<$ $0.001)$ and TWS groups $(p=0.014)$. Interestingly, the TWS group was not found to be significantly different from the WS group ( $p=0.086$ ) in the post hoc tests, but this may indicate lack of power.

A comparison of the individual rats' rates of hindlimb motor recovery (regression slopes of the $\mathrm{AOB}$ recovery trends) indicated that the rates of $\mathrm{AOB}$ recoveries among the three groups were not different $\left(p=0.198, F_{(2,15)}=1.81\right)$. Data are consistent with local spinal plastic changes in the robot rehab framework that led to progressively improving AOB features on the treadmill as a result of the robot-assisted therapy in all groups. However, the AOB regression analysis of the ungrouped $\mathrm{AOB}$ scores and the regressions in the TWS group alone may partly mask differences in the individual sigmoidal AOB changes shown in Figure $3 A$.

\section{Robot body weight support (\%BWS) changes by group}

Figure 5 shows the \%BWS measure changes seen in the three groups of NTWS, TWS, and WS rats. Two-way repeated-measures ANOVA results indicated a strong effect of training. We saw reductions in \%BWS in all groups $\left(F_{(9,135)}\right.$ $=10.73, p<0.001)$. ANOVA results also showed significant difference in the $\%$ BWS response to training among the 3 groups $\left(F_{(2,15)}=3.79, p=0.047\right)$. Post hoc tests with Bonferroni corrections for the group effect showed that the mean \%BWS required for NTWS rats overall was greater than required for TWS and WS rats (though not significantly so, $p=$ 0.150 and $p=0.087$, respectively). However, when the groups were compared daily with a post hoc test (Bonferroni corrections), the differences between NTWS group and the TWS and WS groups were more pronounced and significant $(p<$ $0.05)$, particularly when tested in individual days in the later training phase (Fig. 4), presumably after the large TWS changes had occurred.

There was no interaction effect between the training and group effects, which suggests that the progressive effect of training on \%BWS recovery was not different among the groups. Comparison of individual rats \%BWS regression slopes also indicated that the rates of \%BWS recovery were not different among the three groups $\left(p=0.196, F_{(2,15)}\right.$ $=1.82$ ). In other words, the three groups all reduced dependence on the robot aid at the pelvis at similar rates. The data were again consistent with parallel improvements in each group, with similar slopes of improvements.

The data suggest robot training promotes spinal increases in load bearing capacity through training and voluntary improvements in BWS control. BWS differences between TWS and NTWS may over time contribute to the ability to achieve transition to weight support because robot-supplied BWS were very similar in the TWS and WS rats.

\section{Weight-supported stepping (\%WSS) changes by group}

Percentage WSS was used to assess recovery of autonomous hindlimb weight-supported stepping ability in NTX rats in the regroupings (Fig. 6A,B). Two way repeated-measures ANOVA showed a main effect of training over time $\left(F_{(9,135)}=20.24, p<\right.$ 0.001 ) on $\%$ WSS, indicating that robotic rehabilitation training influenced the \%WSS recovery observed in all groups. However, post hoc testing of the reported training effect showed that mean $\%$ WSS on the last day of training was significantly larger than the first day of training mean \%WSS for only the WS and TWS group ( $p=0.033$ and $p<0.001$, respectively) (Fig. $6 B$ ).

There was also a group effect in this regrouped analysis $\left(F_{(2,15)}\right.$ $=47.01, p<0.001)$, and post hoc test of the reported group effect revealed that the groups were significantly distinct from each other with mean \%WSS for WS rats significantly greater than mean \%WSS for TWS rats, and both WS and TWS rats showing significantly greater \%WSS than mean \%WSS for NTWS rats 
( $p<0.01$ for all comparisons). On the first day of robotic rehabilitation training, the \%WSS in the WS group was significant larger than the NTWS and TWS groups, and this remained so at each time point over the training period $(p<0.05$ for all comparisons between WS group and NWS group at each time point). Conversely, on the first training day, no difference was observed between the relatively low \%WSS scores of the NTWS and TWS groups. However, even by the third training day, there was a significant difference between the TWS and NTWS groups, as the TWS group progressively exhibited significantly more WSS than the NTWS group $(p=0.008)$. As training progressed beyond the third day, the difference between the TWS and NTWS groups progressively increased over time. The TWS group continued to recover and gain more WSS $(p<0.01$ for comparisons with NTWS at all remaining time points) (Fig. 6A).

\section{Rates of gains in \%WSS in the three groups of rats differed}

Two-way repeated-measures ANOVA for \%WSS showed a significant interaction effect between training and group $\left(F_{(18,135)}=\right.$ $5.25, p<0.001)$. This result suggested that the \%WSS improvement patterns seen among the post hoc organized groups were impacted differently by training.

To further evaluate the differences in \%WSS improvement patterns among groups, we analyzed the differences in the individual rates of \%WSS improvement among groups (using linear regression slopes). Figure $7 A$ shows the significantly different rates of improvement in \%WSS in each group. One-way ANOVA comparison of the individual \%WSS regression slopes indicated that the rates of \%WSS improvement were significantly different among the three groups $\left(F_{(2,15)}=26.73, p<0.001\right)$. Post hoc tests also showed that the rate of \%WSS improvement for TWS group was significantly greater than both NTWS $(p<0.001)$ and WS groups $(p=0.005)$. However, the rate of $\%$ WSS improvement in the NTWS and WS groups was not different $(p=0.87)$. This is consistent with the accelerated \%WSS improvement observed in the TWS group shown in Figure 6A. Perhaps there was an acceleration in weight support as rats mastered a coordination of some kind and crossed the "no-man's land" between 25\% and $50 \%$ WSS, as indicated also by the sigmoidal recovery patterns of individual rats. The differences seen in slopes support such an interaction effect (i.e., the influence of training on each group was different).

The results are consistent with TWS rats developing a skill of integrating and improving their support of hindlimb stepping to form functional patterns with forelimbs, trunk, and hindlimb, and thereby crossing the "barrier" between 25\% and 50\%WSS.

\section{WSS rate of improvements and initial WSS}

We sought to determine whether the differences in WSS changes in the post hoc separated and regrouped TWS and NTWS rats related to group differences that were also present before training (and thus predictive of outcome), or whether
Mean \%BWS

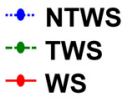

$\rightarrow$ WS
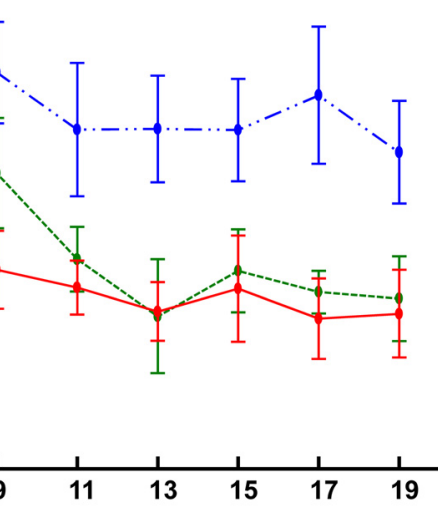

Training Days

Figure 5. Robotic rehabilitation training reduces \%BWS provided by robotic assistance in the WS, TWS, and NTWS groups. Plot repeated-measures ANOVA $\left(F_{(2,15)}=3.79, p=0.047\right)$. * Significant difference between WS and NTWS groups $(p<0.05)$. ${ }^{\#}$ Significant difference between TWS and NTWS groups $(p<0.05)$.

instead they arose from a more mixed continuum of prior abilities. To test this, we related the average rate of WSS improvement to the initial WSS pretraining for all rats (Fig. $7 B$ ). WS rats formed a separated cluster of rates, far from the rest. However, in NTWS/TWS rats, there was a significant linear relationship between the WSS level at outset and rate of WSS improvement (obtained from a linear regression fit to their improvement). In the NWS rats (i.e., combined NTWS and TWS groups together), a linear fit showed an $R^{2}=0.7256$, $(p=0.0001)$. A second-order polynomial fit might also have been suggested by the points, but we found that the additional terms in the fit could not be justified using the Akaike Information Criterion for these data.

These results suggest that an accelerating improvement occurred in WSS in the rats, consistent with the observed sigmoidal transition pattern. It should noted here that the overall aggregate and linear-like \%WSS recovery seen in the TWS group in Figure $6 \mathrm{~A}$ is actually composed of an average of individual sigmoidal $\%$ WSS plots from individual TWS rats, with the fast phase at different times in different TWS rats. Figure $3 C$ shows the sigmoidal \%WSS recovery pattern of a typical TWS rat: an accelerating \%WSS improvement after an initial slow plateau. It is unknown whether the NTWS rats that did not transition would eventually experience such an accelerating improvement if rehabilitation had been further prolonged. We also looked at the initial characteristics of the NWS rats as a whole before training to attempt to predict membership among responders (i.e., in the TWS group) after the fact. We found that, using the initial WSS statistic, we could predict the responders (or TWS rats) with $85 \%$ success, with equal false positives and false negatives if we separated rats by using the midpoint between the mean WSS of NTWS and TWS rats. If instead we sought to avoid false negatives, and ensure rehabilitation was provided to all responders, we could move the boundary down to avoid the false negatives. In this case, we would instead achieve a successful response in rehabilitation in $85 \%$ of the treated rats. 
A

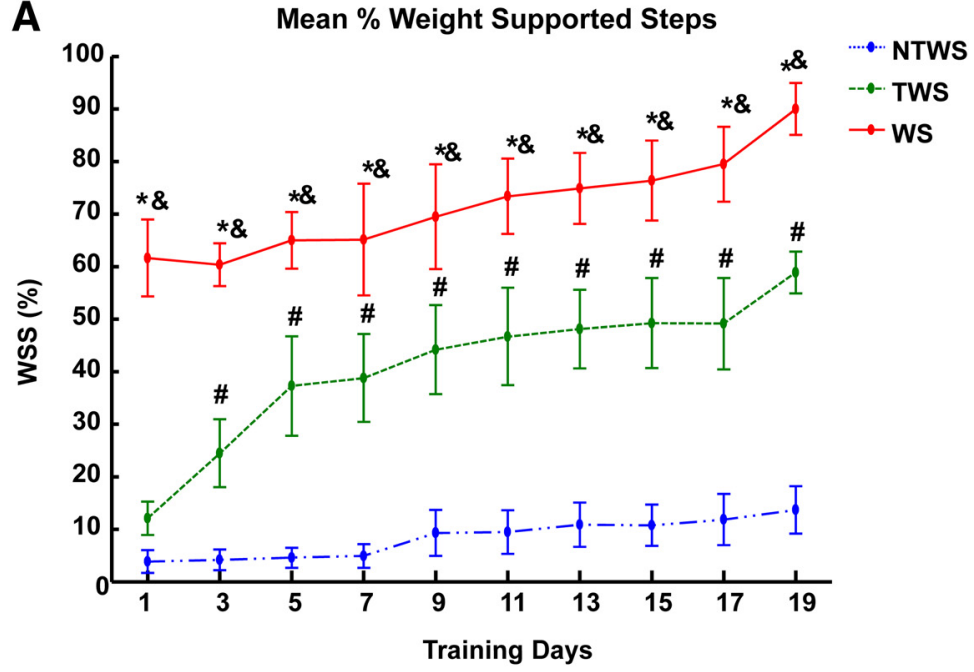

B

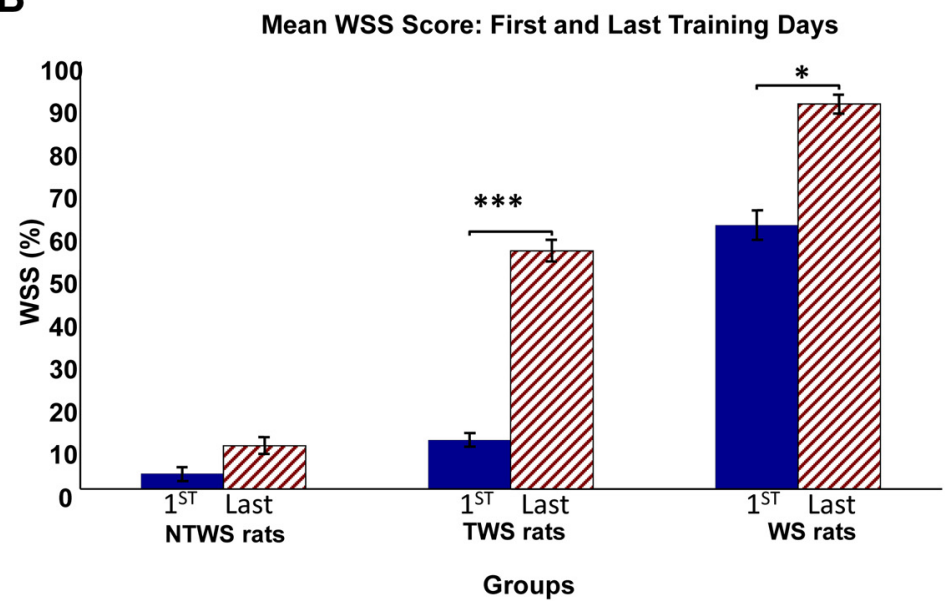

Figure 6. Robotic rehabilitation training increases the proportion of autonomous weight-supported hindlimb stepping (\%WSS) in the WS, TWS, and NTWS groups. A, Plot represents mean \pm SE of \%WSS for each group over the course of robot rehabilitation training. There was a significant training effect (two-way repeated-measures ANOVA, $F_{(9,135)}=20.24, p<0.001$ ) on \%WSS over time in all three groups of rats. There were also differences in the \%WSS recovery performances among the groups as indicated by the group effect reported in two-way repeated-measures ANOVA $\left(F_{(2,15)}=47.01, p<0.001\right)$. Post hoc comparisons of the group effect showed that mean \%WSS for WS group was significantly greater than the TWS group $(p<0.01)$, whereas the mean \%WSS for the NTWS was significantly less than both the WS and TWS groups ( $p<0.01$ for both comparisons). As shown in the figure, this is also evident in the daily post hoc comparisons among groups. *Significant difference between WS and NTWS groups $(p<0.05)$. " Significant difference between WS and TWS groups $(p<0.05)$. "Significant difference between TWS and NTWS groups $(p<0.05)$. B, Post hoc comparisons of the training effect showed that the mean \%WSS on the last training day were significantly greater than the first training day for only the WS and TWS groups of rats $\left({ }^{*} p<0.05\right.$ and ${ }^{* * *} p<0.001$, respectively).

What is learned/gained by rats in the robot rehabilitation training?

We sought to determine whether the rats in the post hoc group that achieved transitions to weight support (TWS rats) had learned to perform fully autonomous locomotion like WS rats. However, we found that none of the TWS rats exhibited weightsupported overground locomotion when off-robot in their cages or on the bench top. In contrast, WS rats walked well in cage, overground, or on treadmill. However, we also tested whether the TWS rats showed any independent \%WSS function gains while on the treadmill. The results of these tests were surprising and instructive, and are shown in Figure 8. We conducted the first sets of these tests in all rats (4 WS, 5 TWS, and 9 NTWS). We inactivated the torque motors of the robot, removing active assistive forces (robot-inactive test). The results are shown in Figure $8 \mathrm{~A}$.
The 5 TWS rats maintained a significant level of weight support after training with the robot motors turned off, although it was significantly decremented from the motors ON condition, (as was also \%WSS in the WS category rats). Rats that were in the TWS group retained high weight support levels even with robot motors off.

In a second, test we then tested rats disconnected from the robot (Off-robot test) but on treadmill. This was only done in a subset of rats (1WS, 3 TWS, 4NTWS; Fig. $8 B$, dotted lines). Similar to the prior observations in cage and overground that we had made in all the TWS rats, the 3 TWS rats tested collapsed back to the pretraining \%WSS.

The relatively small inertia of the passive robot in the robot-inactive test appeared to be a significant feature that the TWS rats may have incorporated into their TWS motor learning on treadmill. It should be noted that even the full WS rats learned to partly rely on (or incorporate and exploit) the robot support, and therefore had some minor but significant deficits in the robot-inactive condition compared with the robot-active condition (Fig. 8A). However, for the 3 TWS rats tested, the loss of the robot inertia was catastrophic. Our data suggest that additional methods will be necessary to achieve full disconnection and autonomy in rats after robot training used here. It still remains to test whether TWS rats could be taught to overcome these issues, and can become weaned off the robot connection, after modified later stage training.

Rats learned features of the robot mechanics and behavior and the context they found themselves in during the rehabilitation. The inertial load was more significant than elastic forces applied for the TWS rats at the trainings end.

\section{Discussion}

\section{Basic observations}

We tested pelvic robotic rehabilitation in adult rats spinalized as neonates (NTX rats). NTX rats, regardless of prior ability, showed significant improvement in hindlimb motor recovery (AOB score), \%BWS, and WSS recovery measures over training. In addition, 5 of the 14 rats classified as NWS before robotic rehabilitation transitioned to a WS classification (TWS rats) while on the active robot. They crossed a "no-man's land" in weight support level that exists between the NWS and WS classifications (Miya et al., 1997; Giszter et al., 1998). Last, TWS rats recovered functions that persisted with robot-inactive, but not with robot disconnected. These data represent the first example of teaching non-weight-bearing spinalized rats the "wheelbarrow race task," which is managed by some NTX rats spontaneously, although learning did not transfer to overground locomotion or off-robot. This difference between inactivating and physically disconnecting the robot was hard to anticipate. 


\section{The robot strategy}

Robot therapy in animal models comprises two variants, based on where the robot interacts: at the leg/ankle or pelvis/trunk. These differ in their kinematic and kinetic effects on locomotor function in the limb. Ankle-based systems interact with the leg during swing, shaping swing kinematics. Within-limb joint kinematics during stance may also alter, constrained by ground contact. Pelvic-based systems leave swing unconstrained but affect trunk kinematics and alter ground reaction forces during stance. These two rehabilitation strategies differ significantly and are only rarely (Y. S. Song and Hogan, 2008) combined. We focused on pelvis-based quadrupedal robot rehabilitation (Giszter et al., 2005; Udoekwere et al., 2006, 2014). Our method differs from others (Dominici et al., 2012) in implanting an osseointegrated orthosis and interacting directly with the skeleton, after the fashion of a clinical halo orthosis. This obviates soft tissue gripping and avoids novel cutaneous and soft tissue deformations during the therapy, which might arise from a sling or sleeve arrangement. Like much human clinical robot therapy, we used an impedance-based assist-as-needed strategy.

Bipedal versus quadrupedal rehabilitation each tests different issues in animal SCI. Bipedal training is used in both partial (van den Brand et al., 2012) and complete spinalinjured rats (Timoszyk et al., 2002, 2005; Cha et al., 2007). In the latter, focus is on enhancing operation of the isolated circuits to improve stepping kinematics and weight support (Dietz et al., 2002; Nessler et al., 2005; See and de Leon, 2013). In partial injury, the emphasis has been to enhance or reveal voluntary control of the hindlimbs (van den Brand et al., 2012). In all cases, robot training has significantly enhanced function. Our quadrupedal study emphasizes both enhancement of stepping mediated by the isolated spinal cord and development of an integrated voluntary control of the trunk and pelvis. NTX rat hindlimb stepping requires none of the pharmacological or electrical stimulation needed after adult injury. We hypothesized that training the two systems in conjunction would provide a path toward autonomous weight support in the NTX rats.

To obtain integrated training of trunk and limb systems, our rehabilitation was applied at the pelvic nexus of limb trunk interaction. Our system likely helped rats to visit mechanical body states they never achieved alone, after having failed to develop autonomous weight bearing earlier. Compliant robot behavior allowed rats to learn trunk-limb interactions essential for weight bearing. The improved hindlimb stepping showed that training allowed spinal systems to discover useful kinematic and kinetic patterns, despite these never being objectively taught or specified at the ankle. The engagement of voluntary and other trunkcoordinative mechanisms supported increases in WSS, and transition of these measures to levels matching autonomously
Rate of Percent Weight Supported Step Recovery with Robot Training

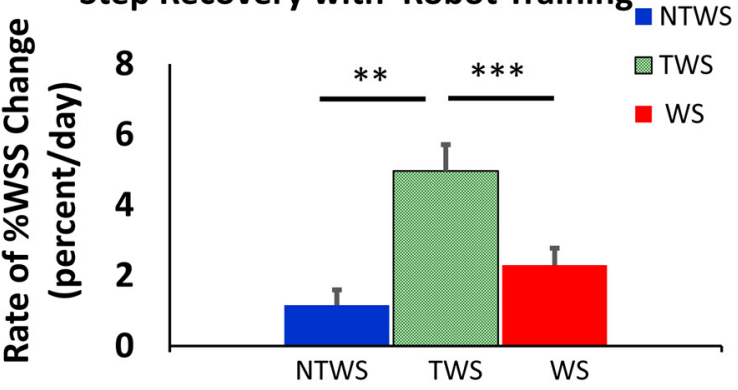

Rate of \%WSS improvement versus Initial WSS

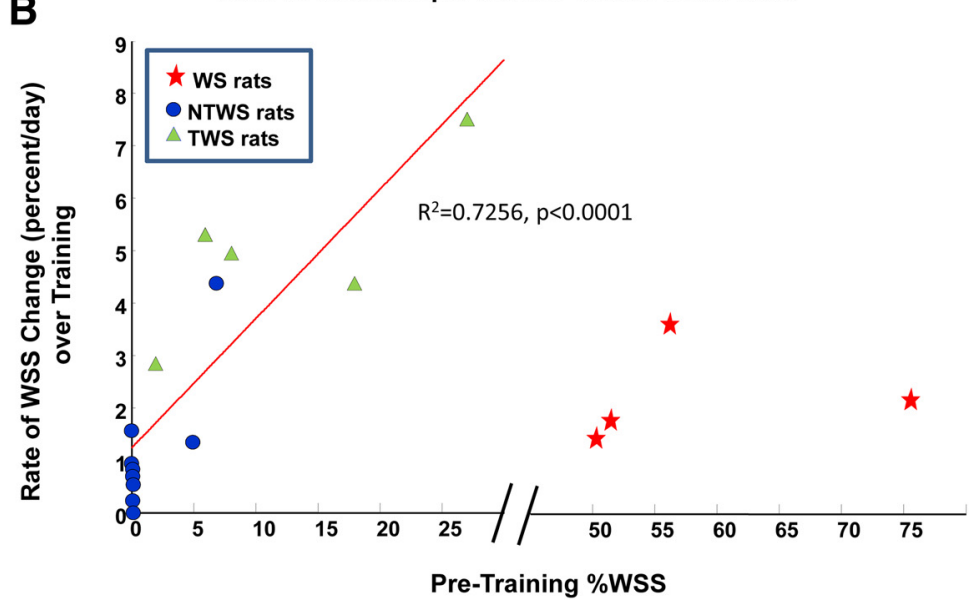

Figure 7. Rates of recovery in autonomous weight-supported hindlimb stepping (\%WSS) with training were different among groups. $A$, The rates of \%WSS recovery (mean $\pm \mathrm{SE}$ ), as determined by the mean regression slopes of the \%WSS recovery trends per group, were significantly different among groups (one-way ANOVA comparison of the \%WSS regression slopes among groups, (2.15) $\left.=26.73,{ }^{* *} p<0.001\right)$. Post hoc tests showed that the rate of \%WSS recovery for the TWS group was significantly greate groups were not different $(p=0.87)$. This observation is consistent with the accelerated \%WSS improvement seen in the TWS group that facilitated transition from a non-weight-supporting classification to a weight-supporting classification with training. $\boldsymbol{B}$, Relationship between rate of \%WSS recovery and \%WSS before training. There was a significant linear relationship between the groups together) $\left(R^{2}=0.726,{ }^{* * * *} p<0.0001\right)$. However, the WS group formed a separated cluster of rates, far from the rest as indicated by the break in the abscissa from $30 \%$ to $45 \%$.

weight-bearing NTX rats, including persistent performance at high levels at the end of training, even with the robot-inactive. Poorer kinematics of stepping likely occurred than in anklebased rehabilitation. However, the greater variability of loading and interaction seen in our system is also consistent with the recognized need for variation and variability in promoting robust rehabilitation (Shah et al., 2012).

\section{The conversion to weight support}

Despite limitations here, $35 \%$ of the NWS rats made quite radical improvements. These responding rats individually demonstrated sigmoidal, accelerating and then saturating improvements in performance, resembling a skill mastery process. As noted, rehabilitation likely conditioned both voluntary and spinal systems, and brought the global variables of pelvis, trunk position, and their interactions with legs into crucial relationships and states needed for learning. All rats recovered significantly, but the remaining $65 \%$ of NWS rats failed to transition to the WS category of classifications. The initial individual level of WSS predicted whether rats were likely to transition. Conceivably, the nonre- 
\%WSS Changes per Group in different conditions

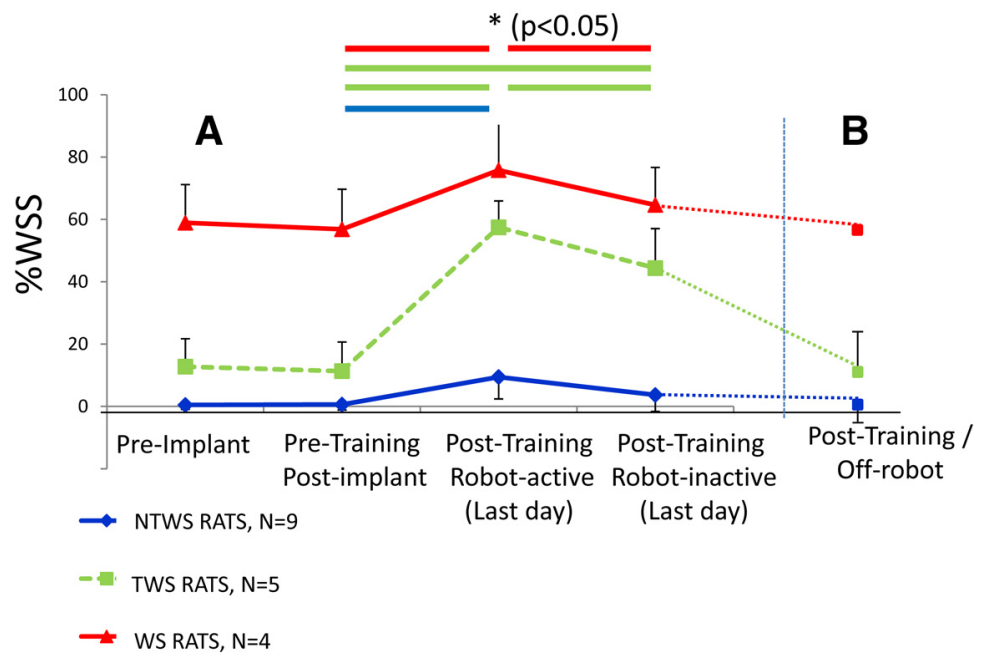

Figure 8. Timeline of changes in weight-supported hindlimb stepping (\%WSS, mean $\pm \mathrm{SE}$ ) over the experiment under different conditions. $A$, All robot-trained rats were tested. Post hoc tests following a two-way ANOVA with repeated-measures analysis showed that the mean \%WSS on the last day of training in the robot-active condition (Post-training/Robot-active) was significantly greater than the pretraining condition for all rat groups. However, the mean \%WSS on the last day of training in the robot-inactive condition (Post-training/Robot-inactive) was significantly less than the robot-active condition in only the WS and TWS rat groups. However, only the TWS group rats had mean \%WSS that was still significantly greater than the pretraining condition. $\boldsymbol{B}$, Fewer rats were tested: 1 WS, 3 TWS, 4 NTWS rats. In the post-training 0ff-Robot condition, the mean \%WSS in the rats tested returned to their pretraining condition values. Horizontal lines between conditions indicate significant differences of $p<0.05\left(^{*}\right)$ for post hoc tests among the conditions in $\boldsymbol{A}$ with all robot-trained rats.

sponding rats might have benefited from pretraining with a legbased therapy (Timoszyk et al., 2002, 2005), pharmacological (Kao et al., 2006), or electrical stimulation intervention (Hsieh and Giszter, 2011; Musienko et al., 2012; Gad et al., 2013). With the means to identify responders and nonresponders described here, it is now feasible in the future to test this. We also speculate that interventions inducing consistent reflex stepping in adultinjured rats (Boyce et al., 2012, 2014; Ziemlińska et al., 2014) might support recovery to levels seen here in neonatal injured animals. Success will depend on similarities between circuits of the adult and NTX spinal cords, the cortical plasticity of adult rats (Oza and Giszter, 2014), and adult spinalized rats' ability to develop the novel coordination seen in NTX rats (Giszter et al., 1998, 2008a, b, 2010).

\section{Spinal and supraspinal roles}

Unlike adult spinalized rats, NTX rats' lumbar cords and cortices developed unconnectedly, which may cause important differences in functions, such as reflex step generation in NTX rats. These differences might enable recovery impossible in adult injury without any additional pharmacological or electrical stimulation interventions.

Changes in both spinal (Petruska et al., 2007) and supraspinal systems likely occurred throughout the therapy. Cortical and map topography changes happen in motor (Oza and Giszter, 2014) and sensory (Aguilar et al., 2010) systems in SCI rats on multiple time scales. Robotic body-weight-support training of motor-incomplete human SCI patients induced plasticity in sensorimotor cortical regions in fMRI (Winchester et al., 2005). Sensory plasticity follows passive motion, bulk repetitive exercise, or skill development in animal SCI models (Kao et al., 2006; Graziano et al., 2013; Moxon et al., 2013). In NTX rats, high weight support achieved autonomously associates with specific motor representations. Good function depends on specific motor areas remaining intact (Giszter et al., 1998, 2008a). In a parallel study (Oza and Giszter, 2015), we have presented cortical changes associated with our robot rehabilitation. In intact adult rats, trunk cortex adapts to novel motor actions and rapidly incorporates them into locomotion mechanics (e.g., in brain machine interface neurorobotics) (W. Song and Giszter, 2011). We therefore speculate that recovery of function in adult rats is unlikely to be constrained by cortical plasticity limits.

Spinal systems responded to pelvic robot rehabilitation by gradually generating better motions, larger ranges of leg joint motion, and patterns of motion and force able to support weight-bearing balanced locomotion with robotic aid. This is consistent with spinal infrastructure for locomotion, which uses local plasticity and "learning" (Jindrich et al., 2009; Edgerton and Roy, 2012; Grau, 2014) and adapts to the rehabilitation "affordances" in support of function. Improved coordination of both the autonomously functioning supraspinal and spinal parts in the divided CNS of NTX rats likely developed during rehabilitation, via the mechanic couplings and by informational exchanges through this mechanics, as well as by improved local functioning of each system when considered alone.

\section{Clinical implications}

Our data relate to issues in clinical rehabilitation and prosthetics. First, NTX rats with low function learned to integrate hindlimb stepping and trunk control to significantly improve function. This suggests that early weight support experience in critical periods, or through development, was not essential to subsequently learn such function. Second, function persisted in the absence of robot actuation (robot-inactive). The rats thus learned to weight support unassisted by an active robot. However, and third, despite this, those rats that gained function failed to exhibit function disconnected from the robot. Motor learning literature suggests that gains in function and skill comprise adaptation, development of internal models, increasing economy of motion, and cognitive and noncognitive contextual framing of these. Our rats may have learned the robot inertia, and incorporated this, either among internal models used, or as context for using the learning and skills achieved during rehabilitation. The rat SCI model may thus be useful to explore adaptation and skill-based gain of function, motor economy, context and dependence on devices during rehabilitation using robots, exoskeletons, and prosthetics. In clinical settings, these various phenomena both contribute to and can potentially confound rehabilitation (FieldFote, 2009; Hornby et al., 2010; Torres-Oviedo et al., 2011; Torres-Oviedo and Bastian, 2012; Krebs and Hogan, 2012, 2013; Finley et al., 2013) and are also fundamental to motor learning more broadly (Kitago and Karakauer, 2013 and see Stanley and Krakauer, 2013).

In conclusion, robot rehabilitation that promotes trunkhindlimb integration promotes significant gain of function in NTX rats that have never walked autonomously. However, most NTX rats did not achieve full autonomy. The route to full auton- 
omy for complete SCI in experiments like these may ultimately depend on promoting better generalization, and broader contexts for the functional skills rehabilitated, and on discouraging learned dependence and economies of motion based on the robotic assistance. However, the high functional plasticity of adult rats demonstrated here is encouraging.

\section{References}

Aguilar J, Humanes-Valera D, Alonso-Calviño E, Yague JG, Moxon KA, Oliviero A, Foffani G (2010) Spinal cord injury immediately changes the state of the brain. J Neurosci 30:7528-7537. CrossRef Medline

Antri M, Orsal D, Barthe JY (2002) Locomotor recovery in the chronic spinal rat: effects of long-term treatment with a 5-HT2 agonist. Eur J Neurosci 16:467-476. CrossRef Medline

Boyce VS, Mendell LM (2014) Neurotrophic factors in spinal cord injury. Handb Exp Pharmacol 220:443-460. CrossRef Medline

Boyce VS, Park J, Gage FH, Mendell LM (2012) Differential effects of brainderived neurotrophic factor and neurotrophin-3 on hindlimb function in paraplegic rats. Eur J Neurosci 35:221-232. CrossRef Medline

Cha J, Heng C, Reinkensmeyer DJ, Roy RR, Edgerton VR, De Leon RD (2007) Locomotor ability in spinal rats is dependent on the amount of activity imposed on the hindlimbs during treadmill training. J Neurotrauma 24:1000-1012. CrossRef Medline

de Leon RD, Acosta CN (2006) Effect of robotic-assisted treadmill training and chronic quipazine treatment on hindlimb stepping in spinally transected rats. J Neurotrauma 23:1147-1163. CrossRef Medline

de Leon RD, Kubasak MD, Phelps PE, Timoszyk WK, Reinkensmeyer DJ, Roy RR, Edgerton VR (2002) Using robotics to teach the spinal cord to walk. Brain Res Brain Res Rev 40:267-273. CrossRef Medline

Dietz V, Curt A (2006) Neurological aspects of spinal-cord repair: promises and challenges. Lancet Neurol 5:688-694. CrossRef Medline

Dietz V, Müller R, Colombo G (2002) Locomotor activity in spinal man: significance of afferent input from joint and load receptors. Brain 125: 2626-2634. CrossRef Medline

Dominici N, Keller U, Vallery H, Friedli L, van den Brand R, Starkey ML, Musienko P, Riener R, Courtine G (2012) Versatile robotic interface to evaluate, enable and train locomotion and balance after neuromotor disorders. Nat Med 18:1142-1147. CrossRef Medline

Edgerton VR, Roy RR (2012) A new age for rehabilitation. Eur J Phys Rehabil Med 48:99-109. Medline

Edwards, AL (1985) Experimental design in psychological research, Ed 5. New York: Harper and Row.

Field-Fote EC (2009) Locomotor training after incomplete SCI: neural mechanisms and functional outcomes. In: Spinal cord injury rehabilitation. Philadelphia: Davis.

Finley JM, Bastian AJ, Gottschall JS (2013) Learning to be economical: the energy cost of walking tracks motor adaptation. J Physiol 591:1081-1095. CrossRef Medline

Fitzgerald J, Fawcett J (2007) Repair in the central nervous system. J Bone Joint Surg Br 89:1413-1420. CrossRef Medline

Gad P, Choe J, Shah P, Garcia-Alias G, Rath M, Gerasimenko Y, Zhong H, Roy RR, Edgerton VR (2013) Sub-threshold spinal cord stimulation facilitates spontaneous motor activity in spinal rats. J Neuroeng Rehabil 10:108. CrossRef Medline

Giszter SF, Kargo WJ, Davies M, Shibayama M (1998) Fetal transplants rescue axial muscle representations in M1 cortex of neonatally transected rats that develop weight support. J Neurophysiol 80:3021-3030. Medline

Giszter SF, Hart CB, Udoekwere UI, Markin S, Barbe C (2005) A real-time system for small animal neurorobotics at spinal or cortical levels. 2nd International IEEE EMBS Conference on Neural Engineering, Arlington, Virginia, pp 450-453.

Giszter SF, Davies MR, Graziani V (2007) Motor strategies used by rats spinalized at birth to maintain stance in response to imposed perturbations. J Neurophysiol 97:2663-2675. CrossRef Medline

Giszter S, Davies MR, Ramakrishnan A, Udoekwere UI, Kargo WJ (2008a) Trunk sensorimotor cortex is essential for autonomous weight-supported locomotion in adult rats spinalized as p1/p2 neonates. J Neurophysiol 100:839-851. CrossRef Medline

Giszter SF, Davies MR, Graziani V (2008b) Coordination strategies for limb forces during weight-bearing locomotion in normal rats, and in rats spinalized as neonates. Exp Brain Res 190:53-69. CrossRef Medline

Giszter SF, Hockensmith G, Ramakrishnan A, Udoekwere UI (2010) How spinalized rats can walk: biomechanics, cortex, and hindlimb muscle scaling-implications for rehabilitation. Ann N Y Acad Sci 1198:279-293. CrossRef Medline

Grau JW (2014) Learning from the spinal cord: how the study of spinal cord plasticity informs our view of learning. Neurobiol Learn Mem 108: 155-171. CrossRef Medline

Graziano A, Foffani G, Knudsen EB, Shumsky J, Moxon KA (2013) Passive exercise of the hind limbs after complete thoracic transection of the spinal cord promotes cortical reorganization. PLoS One 8:e54350. CrossRef Medline

Hogan N, Krebs HI (2004) Interactive robots for neuro-rehabilitation. Restor Neurol Neurosci 22:349-358. Medline

Hornby TG, Reinkensmeyer DJ, Chen D (2010) Manually-assisted versus robotic-assisted body weight-supported treadmill training in spinal cord injury: What is the role of each? PM R 2:214-221. CrossRef Medline

Horst M, Heutschi J, van den Brand R, Andersson KE, Gobet R, Sulser T, Courtine G, Eberli D (2013) Multisystem neuroprosthetic training improves bladder function after severe spinal cord injury. J Urol 189: 747-753. CrossRef Medline

Howland DR, Bregman BS, Tessler A, Goldberger ME (1995) Development of locomotor behavior in the spinal kitten. Exp Neurol 135:108-122. CrossRef Medline

Hsieh FH, Giszter SF (2011) Robot-driven spinal epidural stimulation compared with conventional stimulation in adult spinalized rats. Conf Proc IEEE Eng Med Biol Soc 2011:5807-5810. CrossRef Medline

Jindrich DL, Joseph MS, Otoshi CK, Wei RY, Zhong H, Roy RR, Tillakaratne NJ, Edgerton VR (2009) Spinal learning in the adult mouse using the Horridge paradigm. J Neurosci Methods 182:250-254. CrossRef Medline

Kao T, Shumsky JS, Jacob-Vadakot S, Himes BT, Murray M, Moxon KA (2006) Role of the 5-HT2C receptor in improving weight-supported stepping in adult rats spinalized as neonates. Brain Res 1112:159-168. CrossRef Medline

Kitago T, Krakauer JW (2013) Motor learning principles for neurorehabilitation. Handb Clin Neurol 110:93-103. CrossRef Medline

Krebs HI, Hogan N (2012) Robotic therapy: the tipping point. Am J Phys Med Rehabil 91:S290-S297. CrossRef Medline

Krebs HI, Volpe BT (2013) Rehabilitation robotics. Handb Clin Neurol 110: 283-294. CrossRef Medline

Krebs HI, Hogan N, Aisen ML, Volpe BT (1998) Robot-aided neurorehabilitation. IEEE Trans Rehabil Eng 6:75-87. CrossRef Medline

Krebs HI, Hogan N, Volpe BT, Aisen ML, Edelstein L, Diels C (1999) Overview of clinical trials with MIT-MANUS: a robot-aided neurorehabilitation facility. Technol Health Care 7:419-423. Medline

Krebs HI, Mernoff S, Fasoli SE, Hughes R, Stein J, Hogan N (2008) A comparison of functional and impairment-based robotic training in severe to moderate chronic stroke: a pilot study. NeuroRehabilitation 23:81-87. Medline

Miya D, Giszter S, Mori F, Adipudi V, Tessler A, Murray M (1997) Fetal transplants alter the development of function after spinal cord transection in newborn rats. J Neurosci 17:4856-4872. Medline

Moxon KA, Kao T, Shumsky JS (2013) Role of cortical reorganization on the effect of 5-HT pharmacotherapy for spinal cord injury. Exp Neurol 240: 17-27. CrossRef Medline

Musienko P, Heutschi J, Friedli L, van den Brand R, Courtine G (2012) Multi-system neurorehabilitative strategies to restore motor functions following severe spinal cord injury. Exp Neurol 235:100-109. CrossRef Medline

Nessler JA, Timoszyk W, Merlo M, Emken JL, Minakata K, Roy RR, de Leon RD, Edgerton VR, Reinkensmeyer DJ (2005) A robotic device for studying rodent locomotion after spinal cord injury. IEEE Trans Neural Syst Rehabil Eng 13:497-506. CrossRef Medline

Oza CS, Giszter SF (2014) Plasticity and alterations of trunk motor cortex following spinal cord injury and non-stepping robot and treadmill training. Exp Neurol 256:57-69. CrossRef Medline

Oza CS, Giszter SF (2015) Trunk robot rehabilitation training with active stepping reorganizes and enriches trunk motor cortex representations in spinal transected rats. J Neurosci 35:7174-7189. CrossRef Medline

Petruska JC, Ichiyama RM, Jindrich DL, Crown ED, Tansey KE, Roy RR, Edgerton VR, Mendell LM (2007) Changes in motoneuron properties and synaptic inputs related to step training after spinal cord transection in rats. J Neurosci 27:4460-4471. CrossRef Medline 
Portney LG, Watkins MP (1993) Foundations of clinical research: applications to practice. Norwalk, CT: Appleton Lange.

See PA, de Leon RD (2013) Robotic loading during treadmill training enhances locomotor recovery in rats spinally transected as neonates. J Neurophysiol 110:760-767. CrossRef Medline

Shah PK, Gerasimenko Y, Shyu A, Lavrov I, Zhong H, Roy RR, Edgerton VR (2012) Variability in step training enhances locomotor recovery after a spinal cord injury. Eur J Neurosci 36:2054-2062. CrossRef Medline

Song W, Giszter SF (2011) Adaptation to a cortex-controlled robot attached at the pelvis and engaged during locomotion in rats. J Neurosci 31:31103128. CrossRef Medline

Song W, Ramakrishnan A, Udoekwere UI, Giszter SF (2009) Multiple types of movement-related information encoded in hindlimb/trunk cortex in rats and potentially available for brain-machine interface controls. IEEE Trans Biomed Eng 56:2712-2716. CrossRef Medline

Song YS, Hogan N (2008) Design of an overground interactive therapeutic robot for rodents recovering after spinal cord injury. American Society of Mechanical Engineers Dynamic Systems and Control Conference, Ann Arbor, Michigan, pp 409-411.

Stanley J, Krakauer JW (2013) Motor skill depends on knowledge of facts. Front Hum Neurosci 7:503. CrossRef Medline

Stelzner DJ, Ershler WB, Weber ED (1975) Effects of spinal transection in neonatal and weanling rats: survival of function. Exp Neurol 46:156-177. CrossRef Medline

Timoszyk WK, De Leon RD, London N, Roy RR, Edgerton VR, Reinkensmeyer DJ (2002) The rat lumbosacral spinal cord adapts to robotic loading applied during stance. J Neurophysiol 88:3108-3117. CrossRef Medline

Timoszyk WK, Nessler JA, Acosta C, Roy RR, Edgerton VR, Reinkensmeyer DJ, de Leon R (2005) Hindlimb loading determines stepping quantity and quality following spinal cord transection. Brain Res 1050:180-189. CrossRef Medline

Torres-Oviedo G, Bastian AJ (2012) Natural error patterns enable transfer of motor learning to novel contexts. J Neurophysiol 107:346-356. CrossRef Medline

Torres-Oviedo G, Vasudevan E, Malone L, Bastian AJ (2011) Locomotor adaptation. Prog Brain Res 191:65-74. CrossRef Medline

Udoekwere UI, Ramakrishnan A, Mbi L, Giszter SF (2006) Robot application of elastic fields to the pelvis of the spinal transected rat: a tool for detailed assessment and rehabilitation. Annual International Conference of the IEEE Engineering in Medicine and Biology Society IEEE Engineering in Medicine and Biology Society Conference 1:3684-3687.

Udoekwere UI, Oza CS, Giszter SF (2014) A pelvic implant orthosis in rodents, for spinal cord injury rehabilitation, and for brain machine interface research: construction, surgical implantation and validation. J Neurosci Methods 222:199-206. CrossRef Medline

van den Brand R, Heutschi J, Barraud Q, DiGiovanna J, Bartholdi K, Huerlimann M, Friedli L, Vollenweider I, Moraud EM, Duis S, Dominici N, Micera S, Musienko P, Courtine G (2012) Restoring voluntary control of locomotion after paralyzing spinal cord injury. Science 336:1182-1185. CrossRef Medline

Volpe BT, Krebs HI, Hogan N, Edelsteinn L, Diels CM, Aisen ML (1999) Robot training enhanced motor outcome in patients with stroke maintained over 3 years. Neurology 53:1874-1876. CrossRef Medline

Volpe BT, Krebs HI, Hogan N, Edelstein OL, Diels C, Aisen M (2000) A novel approach to stroke rehabilitation: robot-aided sensorimotor stimulation. Neurology 54:1938-1944. CrossRef Medline

Volpe BT, Krebs HI, Hogan N (2001) Is robot-aided sensorimotor training in stroke rehabilitation a realistic option? Curr Opin Neurol 14:745-752. CrossRef Medline

Volpe BT, Ferraro M, Krebs HI, Hogan N (2002) Robotics in the rehabilitation treatment of patients with stroke. Curr Atheroscler Rep 4:270-276. CrossRef Medline

Volpe BT, Krebs HI, Hogan N (2003) Robot-aided sensorimotor training in stroke rehabilitation. Adv Neurol 92:429-433. Medline

Volpe BT, Ferraro M, Lynch D, Christos P, Krol J, Trudell C, Krebs HI, Hogan N (2005) Robotics and other devices in the treatment of patients recovering from stroke. Curr Neurol Neurosci Rep 5:465-470. CrossRef Medline

Winchester P, McColl R, Querry R, Foreman N, Mosby J, Tansey K, Williamson J (2005) Changes in supraspinal activation patterns following robotic locomotor therapy in motor-incomplete spinal cord injury. Neurorehabil Neural Repair 19:313-324. CrossRef Medline

Ziemlińska E, Kügler S, Schachner M, Wewiór I, Czarkowska-Bauch J, Skup M (2014) Overexpression of BDNF increases excitability of the lumbar spinal network and leads to robust early locomotor recovery in completely spinalized rats. PLoS One 9:e88833. CrossRef Medline 